\title{
锂离子电池硅基负极粘结剂发展现状
}

\author{
王晓钰张渝马蓦 魏良明* \\ (教育部薄膜与微细加工重点实验室 上海交通大学电子信息与电气工程学院微纳电子学系 上海 200240)
}

\begin{abstract}
摘要 在锂离子电池负极材料的研究中, 硅材料以其高达 $4200 \mathrm{mAh} \cdot \mathrm{g}^{-1}$ 的理论比容量, 成为近年来新能源电池领域的 研究热点. 但是在锂化/去锂化过程中, 硅负极体积变化高达 $300 \%$, 导致快速的容量衰减和较短的循环寿命. 目前硅负 极改性最有效的方法之一, 是通过粘结剂来保持活性物质、导电添加剂和集流体间的接触完整性, 减少硅材料在充放 电循环过程中体积变化引起的裂化和粉碎, 保持硅负极的高容量, 提升电池循环性能. 基于硅材料作为锂离子电池负 极的优异特性, 以及目前锂离子电池粘结剂的发展, 将针对锂离子电池硅基负极粘结剂做出系统讨论, 描述不同粘结 剂对电池性能的主要影响, 为锂离子电池硅基负极粘结剂的开发和应用提供研究方向.
\end{abstract}

关键词＼cjkstart锂离子电池; 负极材料; 硅负极; 粘结剂

\section{Recent Development on Binders for Silicon-Based Anodes in Lithium-Ion Batteries}

\author{
Wang, Xiaoyu Zhang, Yu Ma, Lei Wei, Liangming* \\ (Key Laboratory for Thin Film and Microfabrication of the Ministry of Education, Department of Microelectronics and \\ Nanoscience, School of Electronic Information and Electrical Engineering, Shanghai Jiao Tong University, Shanghai \\ 200240, China)
}

\begin{abstract}
In the area of novel power sources, silicon anode in lithium-ion battery, with an ultrahigh theoretical specific capacity of $4200 \mathrm{mAh} \bullet \mathrm{g}^{-1}$, has drawn numerous attentions and got to highlighting spot. Nevertheless, it suffers rapid capacity loss and short cyclability ascribed to the huge volume change during lithiation/delithiation process. So far, one of the most effective methods to ameliorate performances of silicon anode is to modify binders. In this way, the contact integrity among active materials, conductive additives and current collectors can be maintained, which may weaken the cracking and pulverization, keep high specific capacity as well as strengthen the cyclability of silicon anode. Considering both the advantages of silicon anode and the developments of binders, a review on silicon anode in lithium-ion battery will be demonstrated systematically. Besides, we describe the main effects of binders against battery performances. We hope that our review would provide research directions in the developments and applications of binders used in silicon anode of lithium-ion battery.
\end{abstract}

Keywords lithium-ion batteries; anode materials; silicon anodes; binder

\section{1 引言}

目前, 非可再生燃料引起的环境污染问题已成为可 持续绿色发展的重要问题. 为解决严峻的环境污染问 题, 利用清洁能源代替现有的化石燃料, 已经刻不容缓. 在绿色能源行业, 锂离子电池因其高能量储存系统、优 异的电化学能量转换机制、高工作电压、低自放电特性、 重量轻等优异特性, 受到学者与社会的广泛关注. 目前, 锂离子电池已实现工业量产化, 被广泛应用于便携式电 子设备、电动车辆和混合动力电动车辆等热门行业 ${ }^{[1 \sim 6]}$. 但是, 商用锂离子电池负极由传统石墨构成, 电池理论 比容量很低, 仅为 $370 \mathrm{mAh} \cdot \mathrm{g}^{-1}$, 远远不能满足现代社 会对高能量密度电池的需求. 在锂离子电池负极材料的 研究中, 硅材料以其高达 $4200 \mathrm{mAh} \cdot \mathrm{g}^{-1}$ 的理论比容量,
而受到极大关注 ${ }^{[7]}$.

1976 年, 研究人员发现, 锂离子与硅在高温甚至室 温下, 能形成锂硅合金, 开启锂离子电池硅基负极的研 究热潮 ${ }^{[8,9]}$. 硅作为锂离子电池最有前景的负极材料之 一, 有以下优点: (1)高达 $4200 \mathrm{mAh} \cdot \mathrm{g}^{-1}$ 的理论比容量, 远高于传统石墨负极; (2)在锂化/去锂化过程中, 具有极 低电化学电势; (3)高安全性以及环境友好性; (4)高地球 储备量 ${ }^{[10 \sim 18]}$. 但是, 在充放电过程中, 由于锂离子的插 入, 使得硅负极材料的体积膨胀可达 $300 \%$, 从而导致 快速的容量衰减和较短的循环寿命 ${ }^{[19 ~ 24]}$. 导致硅负极 失效的可能原因有以下三种: (1)大的体积变化导致硅颗 粒上产生高内应力，进而粉碎活性物质 ${ }^{[11,25]}$; (2)持续的 体积变化与粉末化过程中, 许多活性材料失去与导电网 络、集流体间电接触，降低电极各材料间的电导率 ${ }^{[22 ~ 24] ; ~}$

\footnotetext{
* E-mail: lmwei@sjtu.edu.cn; Tel.: 13795407683

Received July 16, 2018; published August 31, 2018.

Project supported by the National Natural Science Foundation of China (No. 51272155).

项目受国家自然科学基金(No. 51272155)资助.
} 
(3)体积的不断变化会导致覆盖在电极表面的固体电解 质界面膜(SEI 膜)破裂，并在裸露的硅颗粒表面不断形 成新的 SEI 膜, 消耗电解质中大量锂离子, 并阻止部分 电子传导, 加剧容量衰减 ${ }^{[18,26 ~ 29]}$. 为解决硅负极体积变 化引起的电池循环性能下降的问题, 研究人员有以下几 种改进方式:

第一种是结构改性, 例如制备零维硅纳米颗

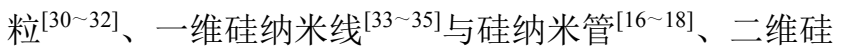
纳米薄膜 ${ }^{[10,36]}$ 等硅负极. 这些纳米结构能够承受锂化/ 去锂化过程中物理应变, 减少体积膨胀导致的裂化和颗 粒粉碎, 增加电极完整性, 最终改善循环特性 ${ }^{[37]}$.

第二种是复合材料改性. 通过硅颗粒与不同材料进 行复合, 制备出性能优异的复合硅负极, 包括硅/碳复合 电极、硅/聚合物复合电极、硅/金属氧化物复合电极等. 利用复合材料缓冲锂化/去锂化过程中引起的体积变化, 减少颗粒粉碎与脱落, 提升电池性能 ${ }^{[38 \sim 44]}$.

第三种是通过高性能粘结剂改性, 这是目前硅负极 改性最简单的方法之一. 通过粘结剂来保持活性物质、 导电添加剂和集流体间的接触完整性, 减少硅材料在充 放电循环过程中体积变化引起的裂化和粉碎, 保持硅负 极高容量, 提升电池循环性能 ${ }^{[37,45]}$.

硅负极材料一般由活性物质硅、导电添加剂与粘结 剂组成, 粘结剂主要用来将所有物质与集流体间紧密粘 结, 在减少活性物质的脱落与粉碎的同时, 防止形成不 稳定的 SEI 膜, 进而保持整个电极的接触完整性, 最终 使电池始终保持较高的容量与优异的循环特性. 性能优 异的硅负极粘结剂应具备以下优点: (1)高均匀性, (2)高 粘附性, (3)强离子/电子传导性, (4)高化学/电化学稳定 性, (5)低膨胀率, (6)低成本与环境友好性, (7)应力缓冲 特性 ${ }^{[19,28,46 \sim 48]}$. 最近研究发现, 在充放电过程中, 一些
聚合物粘结剂的羧基等官能团与硅表面薄二氧化硅氧 化层的羟基能进行共价连接，并在局部破裂时进行重 建，表现出自愈和效应. 这种粘结剂的自愈合特性可持 续修复在充放电过程中造成的损伤，使电池始终保持高 容量和稳定循环性能, 延长复合材料在硅负极中的寿 命 ${ }^{[37,49]}$.

基于硅材料作为锂离子电池负极的优异特性, 以及 目前锂离子电池粘结剂的发展, 我们将针对锂离子电池 硅负极材料常用粘结剂做出系统讨论, 通过不同粘结剂 对硅负极材料的影响和作用机理进行探讨, 为锂离子电 池硅基负极粘结剂的开发和应用提供研究方向.

\section{2 常用粘结剂}

\section{1 聚偏二氟乙烯(PVDF)粘结剂}

在硅负极中, 聚偏二氟乙烯(PVDF) 是最早使用的 粘结剂之一. PVDF, 结构式为 $-\left(\mathrm{CH}_{2} \mathrm{CF}_{2}\right)_{n^{-}}$, 具有对称的 氟键碳骨架, 如图 1a 所示, 溶于 N-甲基-2-吡咯-己酮 (NMP)有机溶剂. PVDF 工作机制如图 1b 所示, 虽然具 有良好的弹性与电化学稳定性, 但 PVDF 与硅颗粒间只 能通过简单的范德华力连接, 并不能支撑在锂化/去锂 化过程中硅负极的大体积变化, 出现明显的脱落与裂化 现象, 如图 1c 所示, 最终导致硅负极的电导率、电池容 量与循环性能急剧下降 ${ }^{[28,46,50 \sim 52]}$.

$\mathrm{Li}$ 等 ${ }^{[53]}$ 利用 PVDF 制备出 $\mathrm{Si} / \mathrm{CB}$ (碳黑)/PVDF 质量 比为 80/12/8 的 PVDF 基硅负极, 并对电极进行热处理. 当温度升高至 $300{ }^{\circ} \mathrm{C}$ 时, 首充比容量达 $2000 \mathrm{mAh} \cdot \mathrm{g}^{-1}$, 并在 50 次循环中保持约 $600 \mathrm{mAh} \cdot \mathrm{g}^{-1}$ 的比容量和 0.170 $\mathrm{V}$ 的低临界电势. 为进一步提高 PVDF 基硅负极的性能, $\mathrm{Xu}$ 等 ${ }^{[54]}$ 利用退火工艺重建并稳定硅颗粒与电极结构, (a)

(b)

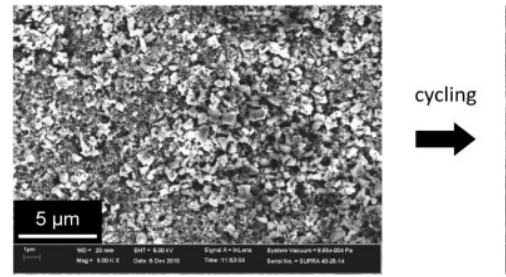

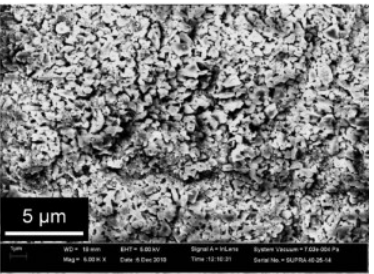

Delithiation

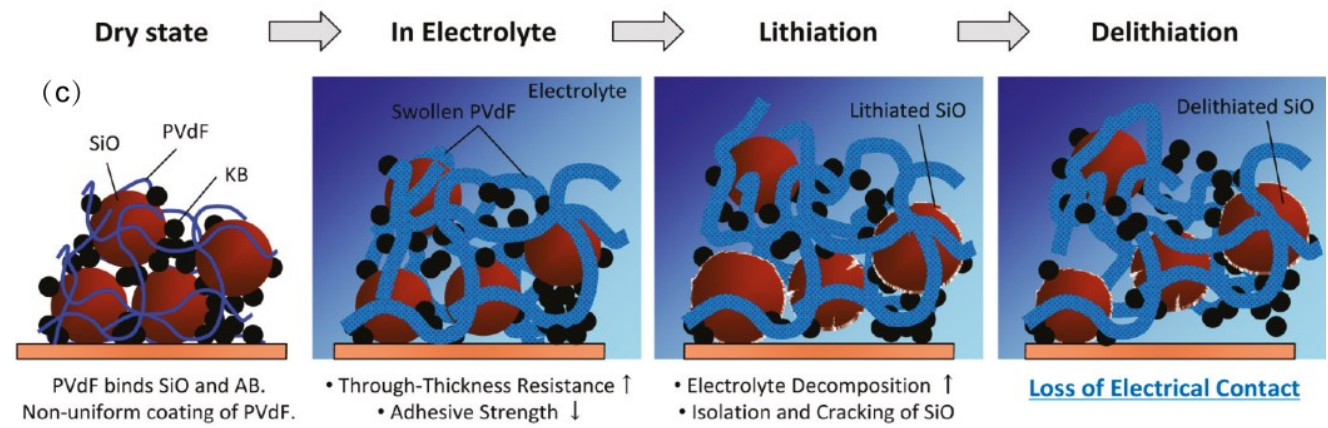

图 1 PVDF 粘结剂[73]

Figure 1 PVDF binder ${ }^{[73]}$

(a) The molecular structure of PVDF binder, the SiO powder composite electrodes with PVDF; (b) SEM images: before (left) and after (right) ten charge/discharge cycles; (c) Schematic illustrations of the proposed mechanism 
获得 $75.6 \%$ 的初始库仑效率和 50 次循环后 $715.6 \mathrm{mAh}$ $\mathrm{g}^{-1}$ 的稳定可逆比容量. Santimetaneedol 等 ${ }^{[55]}$ 建立弹塑性 模型, 在考虑粘结剂应变力的同时, 考虑应力改变的比 率, 准确模拟出 PVDF 基硅负极的力学性能. Grillet 等 ${ }^{[56]}$ 研究发现机械应力对 PVDF 基硅负极循环稳定性 能有显著影响, 在应力环境下进行 160 次循环, PVDF 电 导率下降 45\% 75\%.

\section{2 羧甲基纤维素(钠)(CMC)基粘结剂}

\subsection{1 羫甲基纤维素(钠)(CMC) 粘结剂}

PVDF 与硅颗粒间的弱范德华力连接, 造成 PVDF 基硅负极容量急速下降, 使研究人员急切想寻找可替代 的粘结剂. 2003 年, Drofenik 等 ${ }^{[57]}$ 将羧甲基纤维素钠 (CMC) 作为粘结剂应用于石墨负极, 获得接近石墨理论 比容量的电化学性能. CMC 作为石墨负极粘结剂表现 出的良好性能, 使之成为继 PVDF 后, 引起科学家广泛 关注的粘结剂材料之一. CMC 是纤维素的线性聚合衍 生物, 由 $\beta$ 连接的吡喃葡萄糖残基与羧甲基取代而成, 如图 $2 \mathrm{a}$ 所示, 与硅颗粒间形成稳定的化学连 接 ${ }^{[37,49,58 \sim 60]}$. 与 PVDF 相比, CMC 有以下优点:

(1) $\mathrm{CMC}$ 通过羧基与围绕 $\mathrm{Si}$ 颗粒表面的 $\mathrm{SiO}_{2}$ 薄层 上的羟基进行缩合反应, 产生强共价键, 形成稳定的电 化学连接, 减少锂化/去锂化过程中活性颗粒的脱落与 粉碎, 改善电池循环特性 ${ }^{[59]}$.

（2）虽然 $\mathrm{CMC}$ 具有高刚性与低断裂伸长率 $(5 \%$ $8 \%$ ), 但是循环稳定性优于高弹性 $\mathrm{PVDF}^{[49]}$.

(3) $\mathrm{CMC}$ 与集流体间的粘结强度更大, 在电解质溶 剂中的溶剂吸收率更小 ${ }^{[61]}$.

(4) PVDF 溶剂 NMP 具有毒性, 但 CMC 可直接溶 于水, 是一种对环境友好的粘结剂 ${ }^{[58]}$.

$\mathrm{Li}$ 等 ${ }^{[59]}$ 利用 FTIR 光谱对 $\mathrm{CMC}$ 基硅负极进行化学 分析发现, $-\mathrm{CH}_{2} \mathrm{COOH}$ 基团的数量越多, 循环稳定性越 高. 同时, 相比弹性, $\mathrm{CMC}$ 与活性物质间的化学相互作 用, 是实现更优异循环性能的主要原因. Mazouzi 等 ${ }^{[60]}$ 利用纳米硅与 $\mathrm{CMC}$ 制备出 $\mathrm{Si} / \mathrm{CMC} / \mathrm{CB}$ 复合电极, 在 $\mathrm{pH}$ $=3$ 的酸性介质中, $\mathrm{Si}$ 表面羟基和 $\mathrm{CMC}$ 中羧基酯化反应 更充分, 从而增强 $\mathrm{Si}$ 与 $\mathrm{CMC}$ 间的化学相互作用, 提升 电池循环性能, 最终实现在 $960 \mathrm{mAh} \cdot \mathrm{g}^{-1}$ 的固定比容量 下循环超过 700 次的优异特性.

(a)

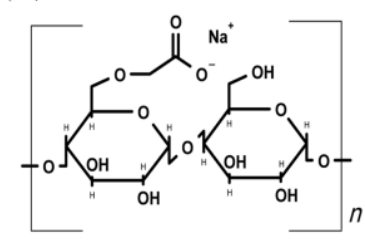

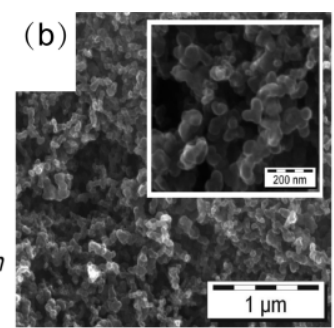

为找出最优质量比, Tarascon 等 ${ }^{[37,62]}$ 进行多组对照 实验发现，当 $\mathrm{Si} / \mathrm{CB} / \mathrm{CMC}$ 质量比为 $1 / 1 / 1$ 时，电极内部 形成最致密结构, $\mathrm{CB} / \mathrm{Si}$ 颗粒完全嵌入电极, 如图 $2 \mathrm{~b}$ 所 示, 内部孔隙率高达 $60 \%$, 能够承受更大的体积变化, 使循环后裂化脱落最少. 此时的复合电极获得最优的电 池循环性能, 如图 2c 所示, 循环 100 次后, 复合电极比 容量高达 $1000 \mathrm{mAh} \cdot \mathrm{g}^{-1}$, 平均库仑效率接近 $99.9 \%$. 随 后, Nguyen 等 ${ }^{[63]}$ 制备出的 CMC 基硅负极, 均获得比 PVDF 更好的循环性能, 很好地体现出 CMC 替代 PVDF 作为粘结剂的优异特性.

考虑到同种粘结剂性能的单一性, 研究人员尝试将 不同的粘结剂混合或交联在一起, 形成新型的复合粘结 剂, 以满足对电池性能多维度的提升.

\subsubsection{CMC-SBR 复合粘结剂}

2006 年, Buqa 等 ${ }^{[64]}$ 将 $\mathrm{CMC}$ 与弹性苯乙烯丁二烯橡 胶(SBR)混合制备出 CMC-SBR 复合粘结剂. SBR 具有高 弹性、强结合力和高耐热性, 与 $\mathrm{CMC}$ 混合后, 能进一步 提升粘结剂性能. 同时, $\mathrm{CMC}$ 与 $\mathrm{SBR}$ 分别可溶于水和乙 酸乙酯等常用无毒或低毒性溶剂, 对未来工业化生产具 有重要意义. 含有 $\mathrm{SBR} / \mathrm{CMC}$ (质量比为 $1 \% / 1 \%$ )复合粘 结剂的纳米硅负极, 以更少量的粘结剂含量实现与 $\mathrm{CMC}$ 相近的初始比容量, 并显现出更优异的循环特性 与更好的电化学性能.

Lee 等 ${ }^{[65]}$ 开发出一种多巴胺功能化肝素/CMC/SBR 的新型聚合物粘合剂, 以更有效地防止 $\mathrm{SiO}_{x}$ /石墨复合 电极的崩解. 利用肝素和 $\mathrm{CMC}$ 间的酸碱相互作用和肝 素中的离子导电磺酸盐基团，以及多巴胺的强粘附性， 使得含有多巴胺-肝素/CMC/SBR 基电极的物理性能比 普通的 $\mathrm{CMC} / \mathrm{SBR}$ 更好，实现在第 150 次循环时具有 $89 \%$ 保留的稳定电池性能. 但是粘结剂本身的比容量很 低, 并不符合社会对越来越高的电池容量密度的需求.

\subsection{3 c-CMC-CA 交联粘结剂}

$\mathrm{Liu}$ 等 ${ }^{[66]}$ 制备出 $\mathrm{CMC}$ 和柠檬酸 $(\mathrm{CA})$ 交联的复合粘 结剂(c-CMC-CA), 与碳/空腔/二氧化硅/硅(CVSS)纳米 球相互连接实现全集成硅负极, 工作机制如图 3a 所示. $\mathrm{CA}$ 中羧基与 $\mathrm{CMC}$ 中羟基在真空、 $150{ }^{\circ} \mathrm{C}$ 下发生缩合 反应，如图 $3 \mathrm{~b}$ 所示，并通过分子链间交联形成酯基，加

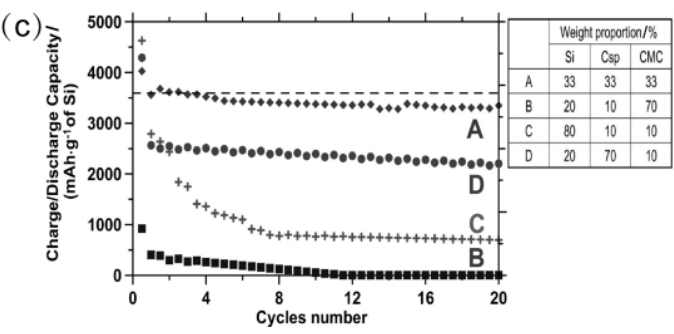

图 $2 \mathrm{CMC}$ 粘结剂[37]

Figure 2 CMC binder ${ }^{[37]}$

(a) The molecular structure of CMC binder, (b) the SEM image before the cycle, (c) the capacity retentions for A, B, C, and D samples. The horizontal dashed line indicates the expected capacity value for full $\mathrm{Si}$ lithiation $\left(\mathrm{Li}_{1} 5 \mathrm{Si}_{4}\right)$. The table inset showing the different coordinates/compositions (wt\%) of the prepared electrodes 

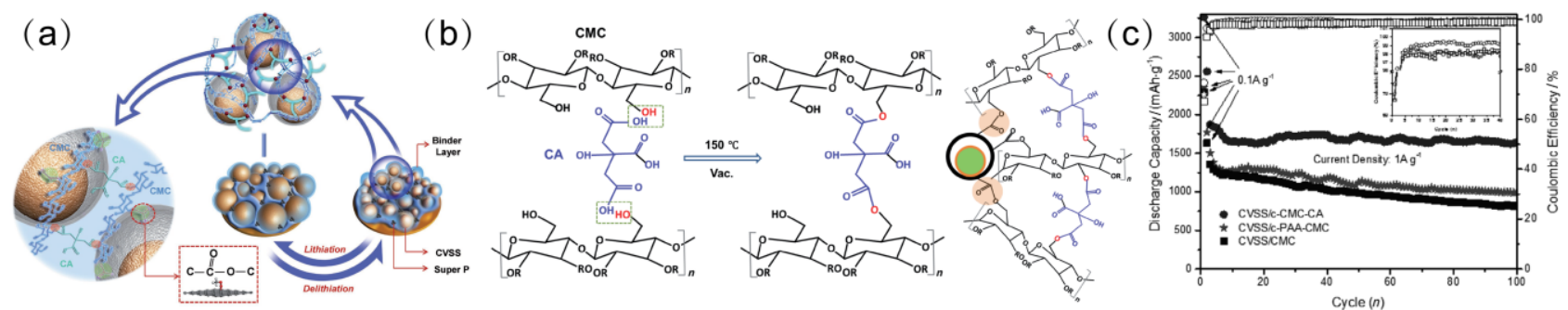

图 3 c-CMC-CA 粘结剂[66]

Figure 3 c-CMC-CA binder ${ }^{[66]}$

(a) Proposed working mechanism of the as-prepared double-shelled-yolk-structured silicon (CVSS) electrode with c-CMC-CA as binder, (b) Cross-linked polymer binder (c-CMC-CA), formed by thermally induced condensation of carboxymethyl cellulose and citric acid, and Chemical bonding between CVSS nanoparticles and c-CMC-CA binder via the esterification between - $\mathrm{COOH}$ groups on the carbon shell and - $\mathrm{OH}$ groups of $\mathrm{CMC}$, (c) Cycling behavior of the CVSS electrode (Coulombic efficiency in inset) with different binders at the current density of $1 \mathrm{~A} / \mathrm{g}$

固复合粘结剂的力学性能. 同时, CMC 中部分游离的羟 基与 CVSS 表面碳壳的羧基间同样进行缩合反应, 在复 合粘结剂和活性 CVSS 纳米球间也形成共价酯键. 两种 酯键与 CVSS 纳米球在电极内部形成牢固的双壳层网状 结构, 有效缓冲充放电循环过程中的大体积变化, 防止 硅粉碎与脱落, 保证电极完整性, 增加高电流密度下的 循环稳定性.

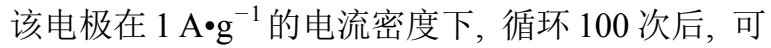
逆比容量高达 $1640 \mathrm{mAh} \cdot \mathrm{g}^{-1}$, 如图 3c 所示. 而且, 在 5 $\mathrm{A} \cdot \mathrm{g}^{-1}$ 的高电流密度下, 1000 次循环后, 容量保持率仍 达 $84.6 \%$, 可逆比容量保持在 $720 \mathrm{mAh} \cdot \mathrm{g}^{-1}$, 为 CMC-CA 粘结剂应用于大电流器件提供了可能性. Karkar 等 ${ }^{[6]]}$ 研究发现, 与聚丙烯酸(PAA)相比, 在低粘 结剂含量下, CMC-CA 的交联效率更好, 接触面密度更 高, 并且 CA 可作为人造 SEI 膜, 帮助稳定电池性能, 使 CMC-CA 基硅负极具有更好的电化学性能 ${ }^{[66]}$.

\section{3 聚丙烯酸(PAA)基粘结剂}

\subsection{1 聚丙烯酸(PAA)粘结剂}

聚丙烯酸(PAA), 分子式为 $\left(\mathrm{CH}_{2} \mathrm{CHOOH}\right)_{n}$, 结构如
图 4a 所示, 可溶于水和乙醇等无毒溶剂. PAA 具有与 $\mathrm{CMC}$ 相似的机械性能, 但 PAA 含有更高浓度的羧基官 能团, 丰富的羧基与硅颗粒形成大量化学键, 增加电极 材料稳定性 ${ }^{[47,59,68]} .2010$ 年, Magasinski 等 ${ }^{[47]}$ 第一次利用 PAA 代替传统粘结剂(PVDF 与 $\mathrm{CMC}$ )制备出复合硅负 极, 在 $\mathrm{C} / 2$ 的电流密度下, 库仑效率高达 $99 \%$, 并且在 超过 100 次循环中保持稳定的性能, 证实粘结剂的低延 展性不会对电池性能产生负面影响, 体现出 PAA 作为 新型粘结剂的优异性.

随后, Shimomura 等 ${ }^{[69-72]}$ 对 PAA 性能进行调制, 利 用 $\mathrm{NaOH}$ 中和 PAA 后作为粘结剂使用. 制备出的硅基复 合电极, 获得具有自组织性能的多孔结构, 有利于缓冲 锂化/去锂化过程中引起的体积变化, 如图 $4 \mathrm{~b}$ 所示, 在 100 次循环中保持高比容量, 初始可逆比容量超过 1000 $\mathrm{mAh} \bullet \mathrm{g}^{-1}$. 中和后的 PAA 可改善 SEI 膜性能，防止电化 学循环过程中电解质的分解, 其基本原理图如图 4c 所 示. Komaba 等 ${ }^{[73]}$ 对 $\mathrm{SiO} / \mathrm{PAA}$ 复合电极进行表征, 探讨 出其工作机理, 如图 4d 所示. 无定形 PAA 聚合物不仅 与 $\mathrm{SiO}$ 颗粒紧密结合而且均匀覆盖, 减少在锂化/脱锂 (a)<smiles>CCC(C(=O)O)C(C)CC(C)(C)C</smiles>

(b) 1500

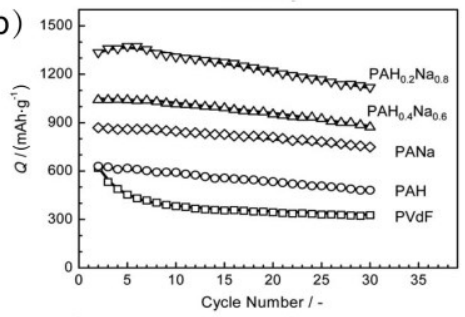

(d) Dry state
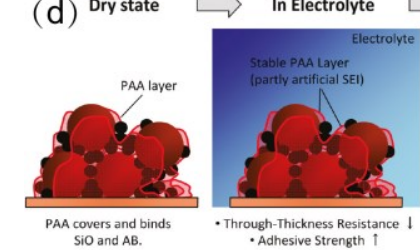

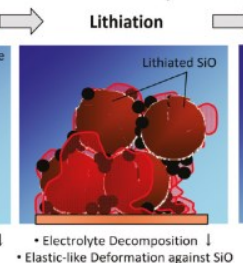

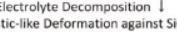

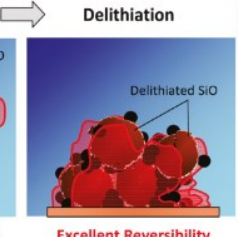

Excellent Reversibility

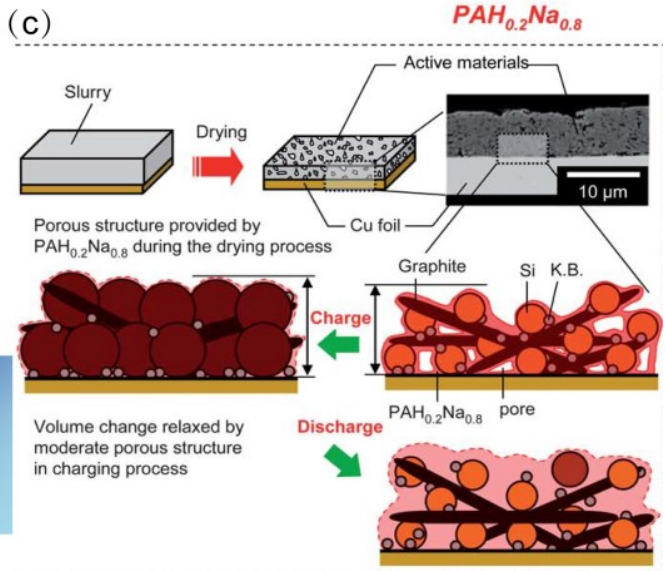

图 4 PAA 和 $\mathrm{PAH}_{0.2} \mathrm{Na}_{0.8}$ 粘结剂

Figure 4 PAA and $\mathrm{PAH}_{0.2} \mathrm{Na}_{0.8}$ binder

(a) the molecular Structure of PAA binder ${ }^{[73]}$. (b) Capacity retention (2nd to 30th cycles) of the Si-graphite electrodes with $10 \mathrm{wt} \% \mathrm{PVdF}_{2} \mathrm{PAH}, \mathrm{PAH} \mathrm{AN}_{0.4} \mathrm{Na}_{0.6}$, $\mathrm{PAH}_{0.2} \mathrm{Na}_{0.8}$, and PANa binders. The electrode loading, including $\mathrm{Si}$, carbon, and binders, was $0.46,0.55,0.40,0.43$, and $0.46 \mathrm{mg} \bullet \mathrm{cm}^{-2}$ for $\mathrm{PVdF}, \mathrm{PAH}, \mathrm{PAH} 0.4 \mathrm{Na}_{0.6}$, $\mathrm{PAH}_{0.2} \mathrm{Na}_{0.8}$, and PANa respectively (PAA are same with PAH) ${ }^{[70]}$. (c) Schematic illustrations of the improved mechanism for the Si-graphite composite electrodes with $\mathrm{PAH}_{0.2} \mathrm{Na}_{0.8}{ }^{[70]}$. (d) Schematic illustrations of the proposed mechanism for the improved cycle ability for the SiO powder composite electrodes with PAA binders $^{[73]}$ 
过程中的大体积变化引起的 $\mathrm{SiO}$ 粉末分离与粉碎, 并有 效抑制复合电极与电解质溶液的溶胀与塌陷.

研究发现, 除氢键外, 通过离子键也可实现与氢键 相似的功能, 使粘结剂在保持电极完整性的同时, 具有 自修复性能, 减小电极容量损失速率. Kang 等 ${ }^{[74]}$ 通过表 面官能化(将硅颗粒与(3-氨基丙基)三甲氧基硅烷(APS) 反应)将胺基共价连接到硅颗粒上, 通过胺基与 PAA 粘 合剂进行动态离子键合, 制备出具有长循环寿命与高可 靠性的硅复合电极. 400 次循环后的复合电极, 比容量 为 $1177 \mathrm{mAh} \cdot \mathrm{g}^{-1}$, 具有 $80 \%$ 的容量保持率.

\subsubsection{PAA-CMC 交联粘结剂}

$\mathrm{Koo}$ 等 ${ }^{[75]}$ 提出一种创新的热固化聚合物粘结剂, 第 一次在实验室中通过化学交联法制备出双聚合物粘结 剂. 利用 PAA 中羧基和 $\mathrm{CMC}$ 中羟基在 $150{ }^{\circ} \mathrm{C}$ 真空下进 行缩合反应生成酯基, 并通过羧酸二聚作用在 PAA 链 中生成酸酐, 制备出具有良好机械性能的 PAA-CMC 三 维交联聚合物粘结剂. PAA 中游离的羧酸与硅颗粒表面 的差基间进行缩合反应, 形成稳定的共价键, 可以有效 地抑制硅纳米颗粒的大幅度移动, 防止循环过程中因大 体积变化而破坏电网，进一步稳定电极结构.

2016 年, 本课题组 ${ }^{[76]}$ 采用自由基接枝聚合法在 $\mathrm{CMC}$ 上接枝聚(丙烯酸钠)(NaPAA), 制备出 NaPAA-g-CMC 共聚物作为硅基负极粘结剂. 实验证实, 由硅/碳黑/粘结剂质量比为 $6 / 2 / 2$ 组成的复合硅负极, 在 $0.45 \mathrm{mg} \cdot \mathrm{cm}^{-2}$ 的硅负载下, 可获得 $2290 \mathrm{mAh} \cdot \mathrm{g}^{-1}$ 的初始 循环比容量. 在 100 次循环后, 比容量保持在 1816 $\mathrm{mAh} \cdot \mathrm{g}^{-1}$, 容量保持率在 $79.3 \%$, 相比其他线性粘结剂, 表现出更好的循环稳定性和更高的库仑效率. 同时, NaPAA-g-CMC 粘结剂具有成本低、易合成和易分离的 优点, 为硅负极材料以及其他电极材料提供出一种非常 有竞争力的解决方案.

\subsubsection{PAA-PVA 交联粘结剂}

Song 等 ${ }^{[77]}$ 通过原位热交联法将水溶性聚丙烯酸 (PAA) 和聚乙烯醇(PVA)进行交联反应, 制备出一种用 于硅负极的新型水溶性聚合物粘结剂, 结构图如图 5a 所示. 这种聚合物粘结剂含有羧基和羟基官能团，可与 硅颗粒表面羟基进行反应，产生强劲的共价结合，形成 具有良好形变特性的聚合物网状结构, 展现出对硅的高 粘附力、高机械强度和自愈合特性，可以有效地适应锂 化/去锂化过程时引起的大体积变化, 改善电极完整性, 从而在高电流密度和活性材料的高负载下，也具有优异 的高库仑效率和循环稳定性. PAA-PVA 基硅负极在 400 $\mathrm{mA} \cdot \mathrm{g}^{-1}$ 的电流密度下, 可获得高达 $3616 \mathrm{mAh} \cdot \mathrm{g}^{-1}$ 的初 始循环比容量和接近 $99.3 \%$ 的平均库伦效率，如图 $5 \mathrm{~b}$ 所 示, 在 100 次循环后剩余可逆比容量仍高达 $2283 \mathrm{mAh}$ $\mathrm{g}^{-1}$ ，显示出 PAA-PVA 基硅负极优异的循环稳定性. 极 高的高库仑效率表明在硅颗粒上具有相对稳定的 SEI 膜，防止一些不可逆的锂储存位点的损失，图 5c 显示的 SEM 图很好地证明这一点. 这也使 PAA-PVA 凝胶粘结 剂在下一代高比容量硅基锂电池中具有很大的应用潜 力 $^{[77]}$.

\subsubsection{PAA-PANI 交联粘结剂}

Lee 等 ${ }^{[78]}$ 将 PAA 与导电聚合物聚苯胺(PANI)结合, 制备出新型聚合物粘结剂 PAA-PANI. PAA-PANI 粘结剂 工作原理如图 5d 所示, 通过掺入 PANI 组分, 使得 PANI 中的胺基通过酸碱反应与 PAA 中羧基相互作用，从而 改善材料机械性能, 有效延缓电极变形, 提高电池循环 稳定性能. 同时, PANI 具有导电能力, 能有效促进硅负 极的电化学反应，提高活性硅的利用率，最终提升电极 容量. 实验证实, 在不同比例的 PANI 掺杂中, PANI 质 量占比为 $10 \%$ 的硅负极显示出最佳的机械性能, 如图 $5 \mathrm{e}$ 所示, 在 300 次循环后比容量保持率可达 $55.8 \%$.
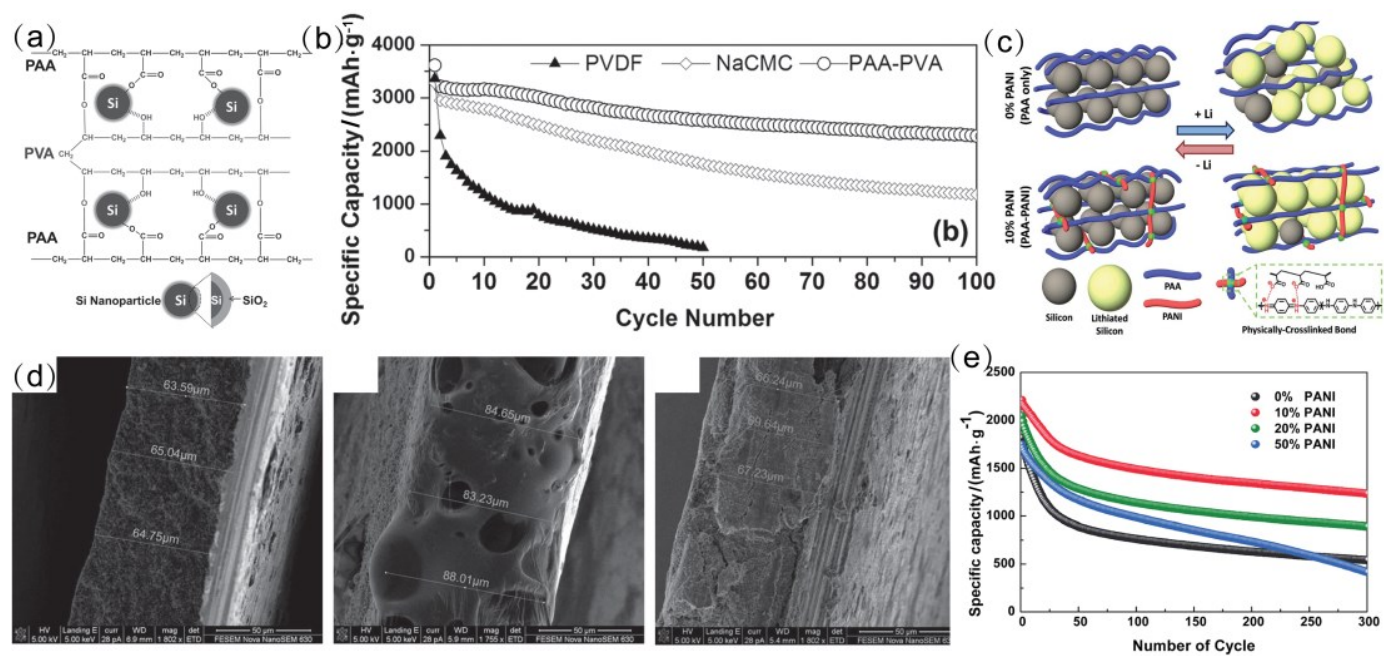

图 5 PAA-PVA 与 PAA-PANI 粘结剂

Figure 5 PAA-PVA and PAA-PANI binders

(a) The chemical structure and illustrative interaction between crosslinked PAA-PVA and silicon particles ${ }^{[77]}$, (b) Coulombic efficiency of Si electrodes with PAA-PVA, NaCMC, and PAA binders ${ }^{[77]}$, (c) design of the PAA-PANI binder ${ }^{[78]}$, (d) Cross-sectional SEM images of the silicon electrodes with PAA-PVA binder before cycling (left), at the end of the 5th discharge (middle) and at the end of the 5th charge (right) ${ }^{[77]}$, (e) Cycle life of LIBs having various compositions of PAA-PANI ${ }^{[78]}$ 


\subsubsection{EDTA-PAA 粘结剂}

除考虑硅负极体积变化引起的容量损失外, 电解质 分解使电解质的成分发生变化, 改变电解质的离子传输 性能. 此外, 生成的 HF 可能会影响硅负极活性材料. Lee 等 ${ }^{[79]}$ 将乙二胺四乙酸(EDTA)引入 PAA 中以抑制 HF 的产生, 并形成稳定的结构与 SEI 膜. 制备出的电极在 $1 \mathrm{C}$ 的电流密度与 300 次循环后容量保留率超过 $83 \%$, 展 示出优异的循环性能.

在以上的三种粘结剂中, PAA 的研究最热, 除上述 以外, 研究人员还利用 PAA 合成聚丙烯酸-嫝基-嘧啶酮 $(P A A-U P y)^{[80]}$ 、聚多巴胺 - 聚丙烯酸 - 聚氧乙烯 $(P D A-P A A-P E O))^{[81]}$ 、聚丙烯酸-聚 (乙二醇-共-苯并咪 唑)(PAA-PEG-PBI ${ }^{[82]}$ 等交联粘结剂, 都取得不错的结 果.

\section{4 小结}

对于常用的粘结剂, PVDF 粘结剂与硅材料间只进 行弱的范德华力连接, 造成电池容量的快速衰减. 而 $\mathrm{CMC}$ 与 PAA 粘结剂与硅材料间共价连接的特性, 在一 定程度上解决容量损失的问题. 但是三种粘结剂结构都 比较简单, 无法有效阻止硅颗粒多方位的脱落. 为解决 这个问题, 研究人员合成复合交联粘结剂, 形成三维粘 结剂结构, 使复合粘结剂与硅表面形成多点相互作用, 有效防止硅颗粒的脱离, 进一步提升电池循环稳定性. 在建立 $3 \mathrm{D}$ 结构的同时, 可以考虑增加具有高弹性、高 导电性能与抑制电解质分解的组分来改善粘结剂的机 械与电化学性能, 在满足稳定性的同时增加容量特性. 但是对于这些常用的粘结剂, 所获得的容量特性远小于 硅的理论比容量, 大多在循环百次后容量减少到 $80 \%$ 以 下，远不能满足商业电池多次充放电稳定使用的特性， 众多研究人员仍在寻找可替代的粘结剂.

\section{3 新型生物高分子粘结剂}

与传统粘结剂相比, 新型生物高分子粘结剂直接从 自然界的天然聚合物中提取，具有环境友好性、丰富性、 低成本、可再生性与多样性等多重优异特性. 不同种类
的新型生物粘结剂中存在丰富多样的基团，能与硅颗粒 表面 $\mathrm{SiO}_{2}$ 上的羟基间进行充分反应，形成稳定强劲的 电化学连接, 为进一步提高循环寿命提供有效的解决方 式.

\section{1 海藻酸盐(Alg)基粘结剂}

\section{1 .1 海藻酸盐(Alg)粘结剂}

2011 年, Kovalenko 等 ${ }^{[83]}$ 第一次利用从褐藻提取出 天然多糖海藻酸盐 (Alg), 混合纳米硅粉, 制备出 $\mathrm{Alg} / \mathrm{C} / \mathrm{Si}$ 复合电极, 如图 $6 \mathrm{a}$ 所示, 在 $1200 \mathrm{~mA} \cdot \mathrm{g}^{-1}$ 的电 流密度下，稳定地循环超过 1300 次，显示出良好的循环 稳定性, 使海藻酸钠成为一种新型的高性能粘结剂. 海 藻酸盐结构如图 $6 \mathrm{~b}$ 所示, 是由 $1 \rightarrow 4$ 连接的 $\beta-D$ 甘露糖 醛酸(M)和 $\alpha-L$-古洛糖醛酸 $(\mathrm{G})$ 残基组成的共聚物, 粘结 剂内部存在大量羧基，可增加电极稳定性，形成稳定的 钝化 SEI 膜, 实现比目前商用的石墨基锂离子电池比容 量高出几倍的电池特性.

海藻酸盐与 $\mathrm{CMC}$ 具有相似的机械性能, 但电化学 性能远优于 $\mathrm{CMC}$, 可能原因为: (1)海藻酸盐中, 羧酸基 才天然存在并均匀分布在聚合物链中，远优于 $\mathrm{CMC}$ 中 羧酸基团随机分布的特性, 因此海藻酸盐形成的电极更 均匀、更高效、更稳定; (2)海藻酸盐比 $\mathrm{CMC}$ 聚合物链 的极性更强, 确保聚合物粘结剂和硅颗粒间具有更好的 界面相互作用，实现电极材料和集流体间更强的粘附 性; (3)达到同样性能所需的海藻酸盐含量更小，可提高 硅材料负载量, 进一步提升电池容量 ${ }^{[83]}$.

\subsection{2 $\mathrm{Ca}^{2+}-\mathrm{Alg}$ 交联粘结剂}

为进一步提升硅基负极电池性能, Liu 等 ${ }^{[84]}$ 在水溶 液中, 将不同藻酸盐链中的 $\mathrm{G}$ 段通过二价阳离子(如 $\mathrm{Ca}^{2+}$ )进行离子交换, 在水中形成稳定交联网络, 如图 $6 \mathrm{c}$ 所示, 即 $\mathrm{Ca}^{2+}-\mathrm{Alg}$ 水凝胶粘结剂. $\mathrm{Ca}^{2+}$ 离子与 $\mathrm{Alg}$ 羧基间 存在离子键, 使 $\mathrm{Alg}$ 分子链由于交联效应而重新排列, 形成更多非晶态结构, 使粘结剂获得更优异的电化学性 能. 制备出的 $\mathrm{Si} / \mathrm{CB} / \mathrm{Ca}^{2+}-\mathrm{Alg}$ 复合电极, 如图 $6 \mathrm{~d}$ 所示, 在 $420 \mathrm{~mA} \cdot \mathrm{g}^{-1}$ 电流密度、 120 次循环后, 获得 1822 $\mathrm{mAh} \cdot \mathrm{g}^{-1}$ 的比容量和 $82.3 \%$ 的容量保持率. (a)

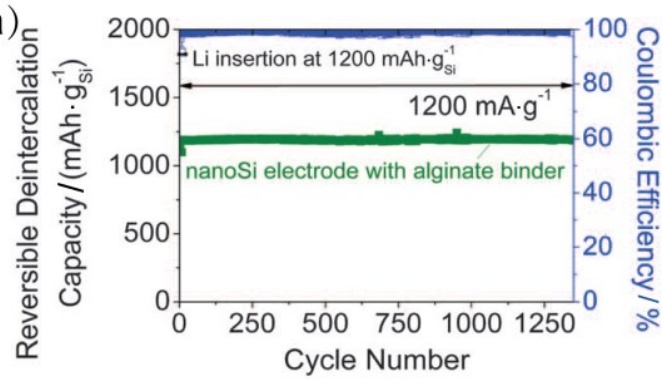

(b)

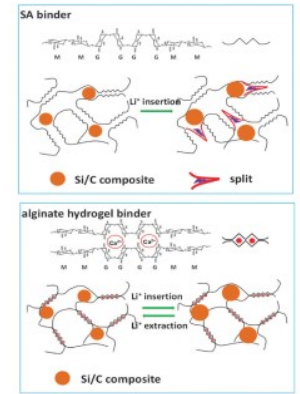

(d)

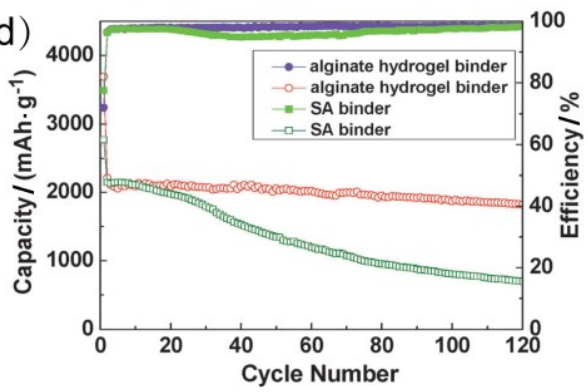

图 $6 \mathrm{Alg}$ 与 $\mathrm{Ca}^{2+}-\mathrm{Alg}$ 粘结剂

Figure $6 \mathrm{Alg}$ and $\mathrm{Ca}^{2+}$-Alg binders

(a) Electrochemical performance of alginate-based nano-Si electrodes (electrode density $=0.50 \mathrm{~g} \cdot \mathrm{cm}^{-3}$, weight ratio of Si/C=3/1) (83] $^{[8 f f e c t}$ of the mechanical integrity of the electrode during the cycling processes: (b) the alginate hydrogel binder ${ }^{[84]}$ and (c) $\mathrm{Ca}^{2+}-\mathrm{Alg}_{\text {binder }}{ }^{[84]}$. (d) Cycle performances of Si/C anodes with pure SA binder and alginate hydrogel binder at $420 \mathrm{~mA} / \mathrm{g}^{[84]}$ 
Yoon 等 ${ }^{[85}$ 利用 $\mathrm{Ca}^{2+}$ 离子介导的 “蛋盒” 结构来静 电交联海藻酸盐, 改善硅负极的韧性和弹性. 改进后的 粘结剂自身具有自愈合特性, 能修复循环过程中的机械 损伤, 延长硅负极的循环寿命的同时增加比容量, 在电 流密度较大、电压平台较小时仍拥有较低的内阻. Zhang 等 ${ }^{[86]}$ 将这种新型粘结剂应用于太阳能电池生产的工业 亚微米硅废料时, 也显示出优异的电池特性, 在 500 次 循环后表现出达 $2522 \mathrm{mAh} \cdot \mathrm{g}^{-1}$ 的比容量和 $76.5 \%$ 的容 量保持率. 除 $\mathrm{Ca}^{2+}$ 以外, $\mathrm{Gu}$ 等 ${ }^{[87]}$ 发现过渡金属阳离子桥 接的所有藻酸盐网络都能够容忍硅的体积变化并有效 地抑制体积膨胀, 而 $\mathrm{Wu}$ 等 ${ }^{[88]}$ 也证实利用不同阳离子对 Alg 进行配位合成优异粘结剂的可行性.

\subsection{3 reDNA-Alg 粘结剂}

此前的粘结剂设计, 主要通过亲水性官能团(即羧 酸、羧酸盐、羟基、胺等)与硅颗粒进行相互作用, 增强 与硅颗粒的粘结性能并构造 3D 网络结构, 以此来保持 电极的完整性. 但是, 这种设计却忽略在电池中占较大 组分的疏水性碳导电剂对电池循环性能的影响. 人体肠 道中的润滑粘液中含有大量的粘蛋白, 是一种高度糖基 化的两亲性蛋白质, 具有分级的刷状结构, 并能完成如 动态收缩、膨胀等与电池体积变化相似的功能.

最近, Kim 等 ${ }^{[89]}$ 受到粘蛋白的抗湿粘附和润滑的启 发, 开发出一种用于硅负极的两亲性粘合剂, 它由热复 性 DNA(reDNA, 即经热处理变性后能在室温下复性的 DNA)和藻酸盐(ALG)组成. reDNA 与 ALG 间进行相互 作用后, ALG 刷从 3D reDNA 框架中伸出, 构成由疏水 性骨架和亲水侧链组成的分级两亲性结构, 同时提供两 亲性和 $3 \mathrm{D}$ 互连结构完整性. reDNA 和 ALG 间的组成比 为 $50 / 50$, 在 $0.5 \mathrm{C}\left(1750 \mathrm{~mA} \cdot \mathrm{g}^{-1}\right)$ 的电流密度下, 在 160 次循环后获得 $80.1 \%$ 的容量保持率. 循环后的 SEM 图表 现出明显的颗粒形态, 其尺寸与循环前相似, 反映出 SEI 并未在循环过程中大量重复生长, 确认界面稳定性, 侧面证实循环性能的稳定性. 虽然 reDNA/ALG 粘结剂 能作为模型案例, 为硅负极提供一种有用的设计原理, 但是在现阶段 DNA 的可行性不高, 要将 DNA 应用在实 际电池上仍有很长的一段路要走.

除此之外, Kong 等 ${ }^{[90]}$ 设计出一种新型多功能结构, 将石墨烯气凝胶(MGA-n)包覆的硅颗粒嵌入由色氨酸 官能化石墨烯量子点(Trp-GQD)、海藻酸钠(Alg)组成的 粘结剂层. Gendensuren 等 ${ }^{[91]}$ 引入聚丙烯酰胺(PAAm)与 Alg 进行化学与物理的双交联来对 Alg 进行改性. 这两 种方式都有效地改善电极的循环性能, 但是却牺牲其高 比容量的特性.

\section{2 壳聚糖(CS)基粘结剂}

\subsection{1壳聚糖(CS)粘结剂}

壳聚糖是一种基于天然碳水化合物的弱碱性天然 聚合物, 是自然界中发现的第二丰富的生物聚合物, 由
随机分布的 $b(1-4)$-连接的 $D$-葡糖胺(脱乙酰单元)和 $N$ 乙酰- $D$-葡糖胺(乙酰化单元)组成, 具有生物可再生性和 生物可降解性等独特特性, 可溶于稀醋酸水溶液 ${ }^{\left[{ }^{[2]}\right.}$. 2010 年, Bridel 等 ${ }^{[37]}$ 尝试使用壳聚糖作为 $\mathrm{Si}$ 负极的粘结 剂, 通过在水中研磨制备出 $1 / 1 / 1$ 质量比的 $\mathrm{CB} / \mathrm{Si} / \mathrm{CS}$ 复 合电极，容量特性一般.

\subsubsection{CS-TPP 交联粘结剂}

2011 年, Ibezim 等 ${ }^{[92]}$ 利用 CS 及其衍生物壳聚糖-三 聚磷酸盐(CS-TPP)与硅进行缩合反应后, 制备出的电极 可获得 $2100 \mathrm{mAh} \cdot \mathrm{g}^{-1}$ 的初始放电比容量, 在 500 次循环 后放电容量保持率高达 $82 \%$, 循环性能稳定, 表明壳聚 糖及其离子交联衍生物可作为较理想的粘合剂, 进一步 提升电池性能.

\subsubsection{C-CS 交联粘结剂}

2014 年, Yue 等 ${ }^{[93]}$ 第一次报道, 利用羧甲基壳聚糖 (C-CS) 作为电池硅负极的水溶性粘结剂. 硅表面极性基 才与 C-CS 表面羟基间形成氢键，在循环过程中可保持 电极的稳定性. SEM 图也证实, 在 40 次循环后, 与 PVDF 大部分粒子脱离表面相比, C-CS 电极表面的活性 材料脱落较少, 在整个电极表面仍然可以发现粘结剂与 硅颗粒大量存在. $\mathrm{Si} / \mathrm{CB} / \mathrm{C}-\mathrm{CS}$ 质量比为 $62 / 30 / 8$ 的复合 电极, 虽然展现出高达 $4270 \mathrm{mAh} \cdot \mathrm{g}^{-1}$ 的首次放电比容 量, 但是循环稳定却很差, 在 $500 \mathrm{~mA} \cdot \mathrm{g}^{-1}$ 速率下, 充放 电循环 50 次后只剩下 $500 \mathrm{mAh} \cdot \mathrm{g}^{-1}$ 的比容量. 为进一步 增加循环特性, $\mathrm{Wu}$ 等 ${ }^{[94]}$ 将海藻酸盐-羧甲基壳聚糖 (Alg-C-CS)交联粘结剂用于硅负极，在 100 次循环后， Alg-C-CS 基硅负极剩余比容量为 $750 \mathrm{mAh} \cdot \mathrm{g}^{-1}$, 虽有一 定的提升, 但是远不能达到商用要求.

\subsubsection{CS-ENR 粘结剂}

最近, Lee 等 ${ }^{[95]}$ 通过常规的过乙酸工艺, 使天然橡 胶得到环氧化，并通过环氧树脂和壳聚糖胺基之间的交 联反应将天然橡胶掺入壳聚糖网络, 设计出具有高粘结 性与高弹性的壳聚糖/环氧化天然橡胶(ENR)粘结剂. 由 于胺基和羟基的存在，壳聚糖可以通过氢键牢固地固定 住硅颗粒，而橡胶部分可以在充放电过程中可逆地拉伸 和收缩, 保持电极完整性. 复合电极在电流密度为 8 $\mathrm{A} \cdot \mathrm{g}^{-1}$ 下循环 1600 次后, 比容量仍为 $1350 \mathrm{mAh} \cdot \mathrm{g}^{-1}$, 在 $1 \mathrm{~A} \cdot \mathrm{g}^{-1}$ 的电流密度下循环 500 次后, 比容量仍达 2300 $\mathrm{mAh} \cdot \mathrm{g}^{-1}$, 显示出 CS-ENR 粘结剂非常好的循环特性, 以及可以在大电流下工作的可能性.

\section{$3.3 \beta$-环糊精聚合物( $\beta-\mathrm{CDp}$ )基粘结剂}

\subsection{1 $\beta-\mathrm{CDp}$ 粘结剂}

$\beta$-环糊精 $(\beta-\mathrm{CD})$ 是葡萄糖作为单体单元的 7 元糖大 环化合物, 容易通过酶促过程直接从普通淀粉中合成. 强碱性条件下，利用表氯醇(EPI)进一步官能化，能使 $\beta$ - CD 形成具有各种羟基和理想骨架的超支化 $\beta$-环糊精 聚合物 $(\beta-\mathrm{CDp})$, 如图 7a 所示. 
$\beta-\mathrm{CDp}$ 的超支化网络结构呈现出与硅的多维氢键 相互作用, 提供两者间的牢固接触, 形成稳定的电极. 在锂化/去锂化过程中, 如图 $7 \mathrm{~b}$ 所示, 这种稳定的电极 多维网络结构能使部分与粘结剂失去接触的硅颗粒在 其他位点恢复与超支化粘结剂网络的相互作用, 产生自 愈合效应, 减少比容量损失, 显著提升电池循环性能. 同时, $\beta-\mathrm{CDp}$ 不溶于碳酸脂基电解质溶剂, 在电解质中 具有很高的稳定性 ${ }^{[96,97]} .2014$ 年, Jeong 等 ${ }^{[97]}$ 首次引入 $\beta-\mathrm{CDp}$ 作为新型硅负极粘结剂, 制备出 $\mathrm{Si} / \beta-\mathrm{CDp}$ 质量比 为 $3 / 1$ 的复合电极, 在 $1 \mathrm{C}$ 条件下循环 150 次, 如图 $7 \mathrm{c}$ 所示, 具有 $50.6 \%$ 的容量保持率.

\subsubsection{Alg- $\beta$-CDp 交联粘结剂}

利用聚合物交联特性, 能进一步改进粘结剂的性 能, 提升硅基负极的电化学性能. Kwon 等 ${ }^{[97]}$ 将超支化 $\beta-\mathrm{CDp}$ 与不同质量比的一维海藻酸盐混合制备出不同 粘结剂. $\mathrm{Alg}-\beta-\mathrm{CDp}$ 工作原理图如图 $7 \mathrm{~d}$ 所示, 一方面, $\beta-\mathrm{CDp}$ 会通过分子内氢键形成聚集体, 添加海藻酸盐可 减少 $\beta-\mathrm{CDp}$ 的聚集而不损害其优异特征, 另一方面, $\beta-\mathrm{CDp}$ 可以中和海藻酸盐中羧酸酯单元间的静电反应, 使电极材料中聚合物分布得更加均匀. 当海藻酸盐占比 为 $13 \%$ 时, 电极显示出最佳的循环性能, 如图 7e 所示, 在 100 次循环后，仍保持 $70.6 \%$ 的容量保持率.

\subsection{3 $\beta$-CDp-6AD 交联粘结剂}

除静态交联外, Kwon 等 ${ }^{[98]}$ 利用超支化 $\beta$-环糊精聚 合物和包含六个金刚烷单元的树枝状没食子酸交联剂 (6AD) 间的动态相互作用制备出超分子交联聚合物粘结 剂.

与传统的共价交联不同, 动态交联的可逆性使得聚 合物链间均匀交联, 并具有自愈和效应, 如图 8a 所示, 实现粘结剂与硅的密切相互作用, 形成稳定的电极膜结 构和可控制的电极-电解质界面, 克服硅在循环过程中
引起的体积变化, 保持电极完整性, 最终提高循环性能, 增强循环寿命. 实验制备的 $\beta$-CDp-6AD 基硅负极, 如图 $8 \mathrm{~b}$ 所示, 在约 $0.8 \mathrm{mg} \cdot \mathrm{cm}^{-2}$ 硅负载、 $8.5 \mathrm{wt} \% 6 \mathrm{AD}$ 占比的 实验条件下, 经过 150 次循环后, $\beta-\mathrm{CD} / \mathrm{AD}$ 质量比为 $1 / 0.5$ 时, 容量保持率可达 $90 \%$, 实现电池循环性能的大 的提升 ${ }^{[98]}$.

\section{4 生物胶聚合物粘结剂}

\subsection{1阿拉伯胶(GA)粘结剂}

GA 在自然界中广泛存在，由具有丰富羟基的多糖 与富含多肽侧链的糖蛋白组成，是一种低成本，在自然 界中丰富存在, 且对环境无害的双功能天然聚合物粘结 剂. 多糖具有长碳水化合物单糖单元，富含羟基，使粘 结剂与硅颗粒间连接紧密. 糖蛋白富含长寡糖链, 共价 连接于多肽侧链, 呈现出长螺旋状结构, 与混凝土中纤 维相似, 表现出低模量、高伸长率的特性, 增加电极的 机械耐受性, 减少体积变化引起的容量损失 ${ }^{[99]} .2014$ 年, Ling 等 ${ }^{[99]}$ 第一次将 GA 作为粘结剂应用于硅负极, 在 $1000 \mathrm{mAh} \cdot \mathrm{g}^{-1}$ 的比容量条件下, 循环超过 1000 次, 表现 出极其优异的长期循环稳定性.

Zhao 等 ${ }^{[100]}$ 利用 GA 与 PAA 混合制出低成本的复合 粘结剂, 成功制备出有弹性、防裂性能优异的硅负极. GA-PAA 复合结构如图 9a 所示, GA 中丰富的羟基与 PAA 中羧酸基团间能进行酯化反应, 建立柔韧网络, 增 强机械强度, 改善界面连接性和结构完整性. 同时, 由 酯化反应产生的水蒸气在复合电极材料内部形成微米 孔隙, 减少应力带来的损失, 阻止裂纹的形成和传播, 提高电池的循环性能. 硅/碳/粘结剂质量比为 $2 / 1 / 1$ 时, 电极表现出优异的电化学性能, 比容量高达 2890 $\mathrm{mAh} \cdot \mathrm{g}^{-1}$, 如图 $9 \mathrm{~b}$ 所示, 在 $1000 \mathrm{mAh} \cdot \mathrm{g}^{-1}$ 条件下获得充 电放电循环超过 1000 次的优异循环特性. (a)
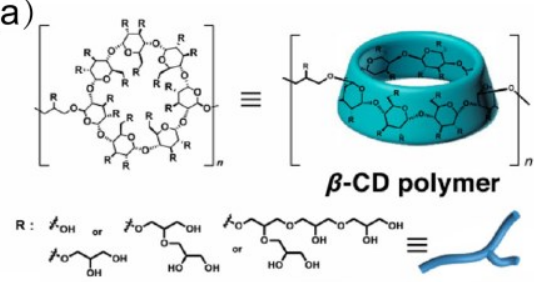

(C) $\beta-C D p$

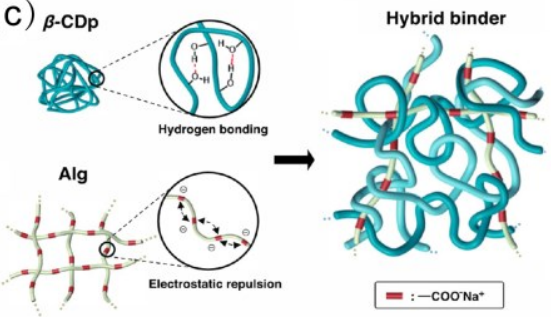

(b)

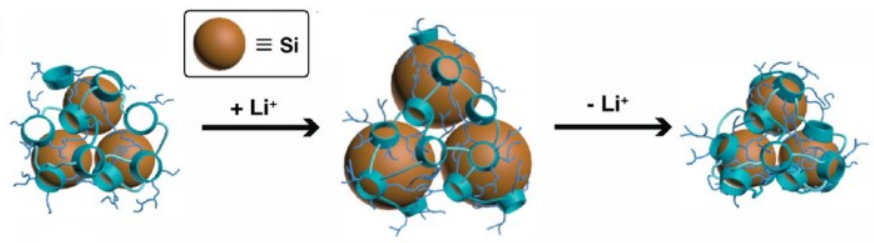

(d)

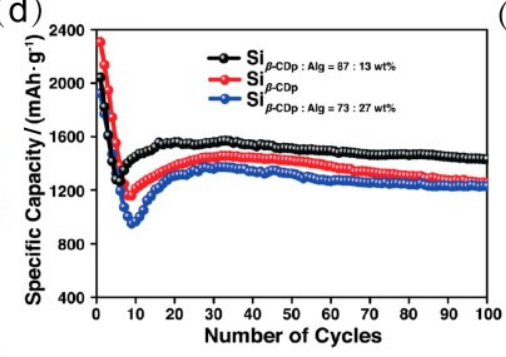

(e)

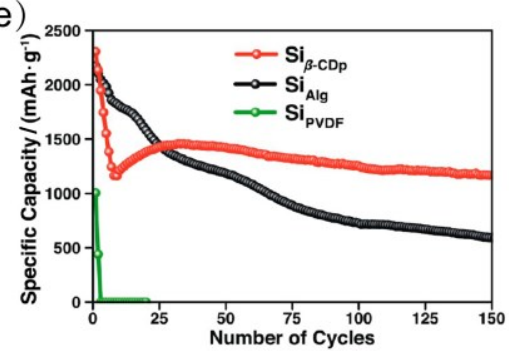

图 $7 \beta-\mathrm{CDp}$ 与 $\beta$-CDp-Alg 粘结剂 ${ }^{[97]}$

Figure $7 \quad \beta$-CDp and $\beta$-CDp-Alg binders ${ }^{[97]}$

(a) Structural formulas and graphical representations of $\beta$-CD polymer $(\beta$-CDp) binder. (b) Schematic representations of Si-binder configurations with $\beta$-CDp binder during lithiation/delithiation, (c) Proposed mechanism of synergistic effect in the $\beta$-CDp/Alg hybrid binder, (d) The cycle performance of the Si electrodes based on a hybrid binder approach employing different ratios of $\beta$-CDp and Alg. The electrode compositions were $\mathrm{Si} / \mathrm{Binder} / \mathrm{SuperP}=60 / 20 / 20$ by weight, and the loading amounts of $\mathrm{Si}$ were $0.6 \mathrm{mg} \cdot \mathrm{cm}^{-2}$, (e) Comparison of the cycle performance of $\mathrm{Si}_{\beta-\mathrm{CDp}}$ with others when measured at $1 \mathrm{C}$ 

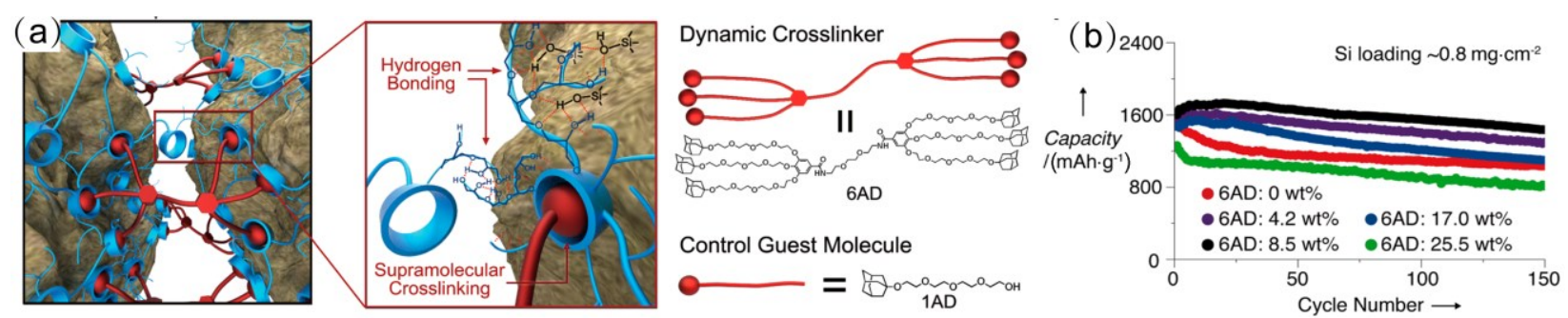

图 $8 \beta$-CDp-6AD 粘结剂[98]

Figure $8 \quad \beta$-CDp-6AD binder ${ }^{[98]}$

(a) Proposed working mechanism of dynamic cross-linking of $\beta$-CDp and 6AD in an electrode matrix, (b) Cycling performance of Si electrode based on the different amounts of $6 \mathrm{AD}(0 \sim 25.5 \mathrm{wt} \%)$ at $0.5 \mathrm{C}\left(1500 \mathrm{~mA} \cdot \mathrm{g}^{-1}\right)$, the ratio of components was fixed as $\mathrm{Si} / \mathrm{SuperP} / \mathrm{binder}=60 / 20 / 20 \mathrm{wt} \%$. Si mass loading $=c a .0 .8 \mathrm{mg} \bullet \mathrm{cm}^{-2}$
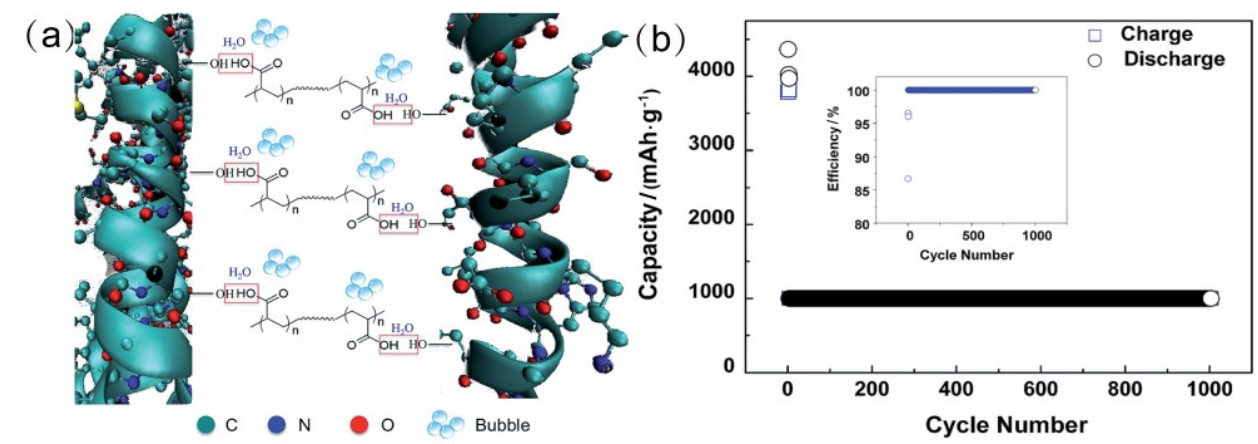

图 9 GA-PAA 粘结剂[100]

Figure 9 GA-PAA binder ${ }^{[100]}$

(a) Schematic diagram of the polymeric reaction: the generation of water and formation of the crack-blocking GA-PAA composite binder, (b) Reversible Li-extraction capacity and Coulombic efficiency of the Si electrodes for the Li insertion level fixed to $1000 \mathrm{mAh} \cdot \mathrm{g}^{-1}$ at $1 \mathrm{C}$ rate $\left(4200 \mathrm{~mA} \cdot \mathrm{g}^{-1}\right)$ over the potential window of $0.01 \sim 1 \mathrm{~V}(\mathrm{Si} / \mathrm{C} /$ binder $=2 / 1 / 1, \mathrm{wt} \%)$

\subsection{2 瓜耳胶(GG)与刺槐豆胶( $(\mathrm{BBG})$ 粘结剂}

生物聚合物瓜尔胶 $(\mathrm{GG})$, 由瓜尔豆种子产生, 由具 有 $(1 \rightarrow 6)$ 键连接的 $\alpha-D$-吡喃半乳糖单元的线性 $(1 \rightarrow$ 4)- $\beta$ - $D$-甘露吡喃糖基单元组成, 如图 10a 所示, 是一种 低成本、高环保性和具有生物可再生性的多糖聚合物。 $\mathrm{GG}$ 分子中含有大量的极性羟基, 能提供更多的连接位 点, 与硅形成稳定共价键, 减少硅粒子与电解质接触, 提高电极内导电网络的稳定性. 同时, $\mathrm{GG}$ 与聚环氧乙 烷固体电解质性质类似, 可将锂离子更有效地转移到硅 表面, 转移机制如图 $10 \mathrm{~b}$ 所示, 有效降低电极极化, 进 一步改善容量与循环特性 ${ }^{[101,102]}$

2015 年, $\mathrm{Liu}$ 等 ${ }^{[101]}$ 首次利用瓜耳胶粘结剂制备出复 合硅负极, 在 $2100 \mathrm{~mA} \cdot \mathrm{g}^{-1}$ 的电流密度下, 可获得高达 $3364 \mathrm{mAh} \cdot \mathrm{g}^{-1}$ 的初始放电比容量, 并在 300 次循环后比 容量达到 $1561 \mathrm{mAh} \cdot \mathrm{g}^{-1}$, 如图 10c 所示. 更可贵的是, 将电池放电容量限制在 $1000 \mathrm{mAh} \cdot \mathrm{g}^{-1}$, 能稳定循环至 930 圈, 如图 $10 \mathrm{~d}$ 所示. Kuruba 等 ${ }^{[103]}$ 也证明 $\mathrm{GG}$ 是一种 极有前景的粘结剂.

刺槐豆胶 $(\mathrm{LBG})$ 与瓜尔胶 $(\mathrm{GG})$ 相似, 改变半乳糖侧 链在甘露糖骨架中分布量可获得 $L B G$, 但由于 GG 中亲 水半乳糖基团的增加, 使 $\mathrm{GG}$ 与硅的天然氧化物层结合 更充分, 形成更稳定的复合电极, 所以 $\mathrm{GG}$ 粘结剂电化 学性能优于 LBG 粘结剂[102].

\subsection{3 黄原胶粘结剂 $(\mathrm{XG})$}

天然黄原胶粘结剂 $(\mathrm{XG})$ 是一种多糖粘结剂, 基础 结构如图 11a 所示, 具有与纤维素相同的骨架, 由 $\beta$ - $D$ 甘露糖-(1,4)- $\beta$ - $D$ - 葡糖醛酸- $(1,2)-\beta$ - $D$ - 甘露糖构成的三 糖侧链, 这些侧链与 C-3 骨架中的其他葡萄糖残基连接 在一起.

其中，主骨架具有疏水性，并且能在一系列分子间/ 分子内利用氢键进行相互作用, 形成双螺旋超结构. 虽 然位于三糖侧链上的带电基团由于库仑排斥作用而不 利于这种缠结过程，使得侧链部分脱离螺旋骨干，但是 附着在骨架上的甘露糖残基被乙酰化，使得螺旋骨架的 末端基团能促进超结构进行自组装, 并与相邻骨架相互 作用, 从而在两个不同的骨架中继续形成螺旋结构, 最 终形成比 $\mathrm{Na}-\mathrm{CMC}$ 与海藻酸盐更坚硬、更稳定的黄原胶 双螺旋超结构粘结剂. 剥离实验中, XG 基硅负极机械 剥离后脱落更少, 进一步证实 XG 粘结剂的稳定性. 从 结构上看, $\mathrm{XG}$ 双螺旋超结构与千足虫相似, 与活性材 料具有较多的活性位点. 从化学角度看, 黄原胶粘结剂 每个侧链具有离子-偶极相互作用, 形成类似千足虫腿 上粘附垫的结构, 如图 $11 \mathrm{~b}$ 所示, 创造出极强的粘附 性 ${ }^{[104,105]}$.

Chen 等 ${ }^{[104]}$ 第一次利用 XG 粘结剂制备出硅/石墨 烯/粘结剂复合电极, 并获得优异的循环性能与倍率能 力. 随后, Jeong 等 ${ }^{[105]}$ 利用天然黄原胶双螺旋超结构粘 

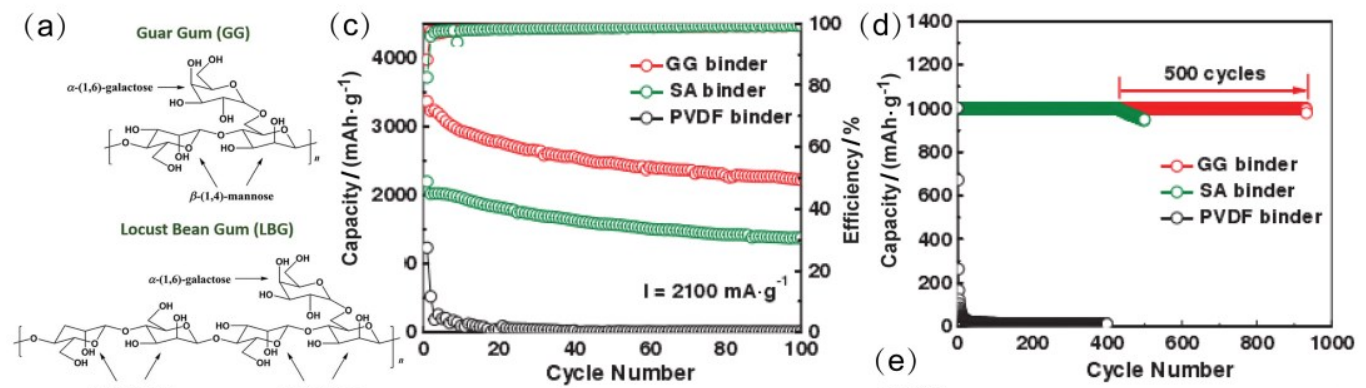

(b)
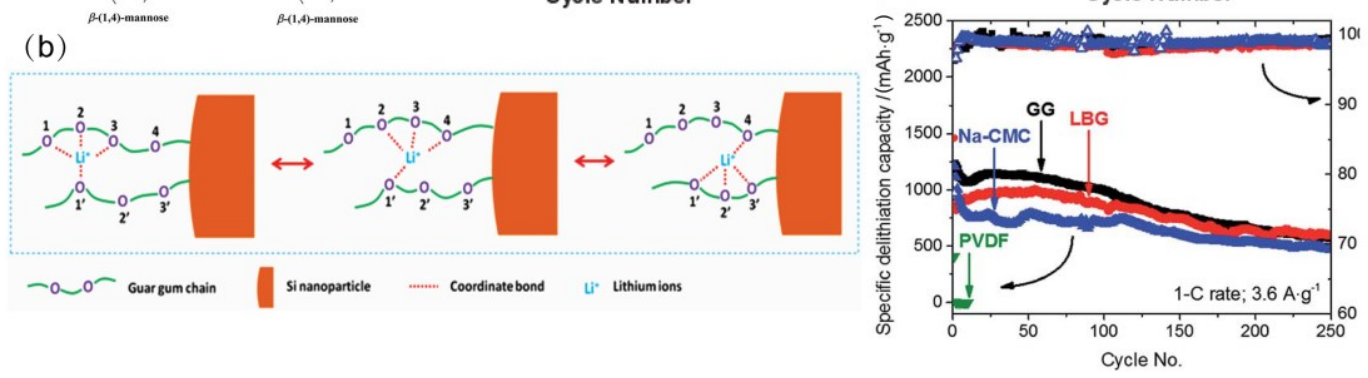

图 $10 \mathrm{GG}$ 与 $\mathrm{LBG}$ 粘结剂

Figure 10 GG and LBG binders

(a) The chemical composition of guar gum $(\mathrm{GG})$ and locust bean gum $(\mathrm{LBG})^{[102]}$, (b) Schematic illustration of lithium-ion transfer in the GG binder ${ }^{[101]}$, (c) Electrochemical performance of SiNP anodes with different binders at $2100 \mathrm{~mA} \cdot \mathrm{g}^{-1}$ between 0.01 and $1.2 \mathrm{~V}^{[101]}$, (d) cycle performance with limited discharge capacity of $1000 \mathrm{mAh} \cdot \mathrm{g}^{-1}$ at $1000 \mathrm{~mA} \cdot \mathrm{g}^{-1[101]}$, (e) The symmetrical cycling performance of SiNPs with GG, LBG, Na-CMC and PVDF binders ${ }^{[102]}$

(a)

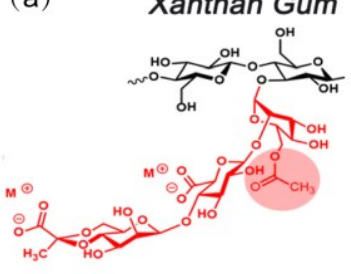

(b)

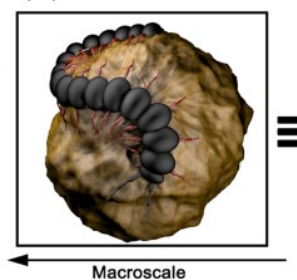

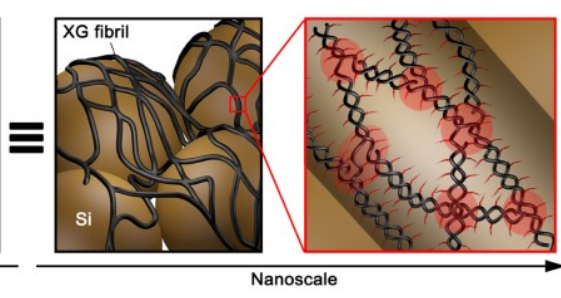

(c)

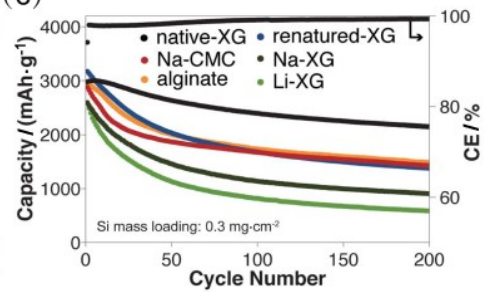

图 $11 X G$ 粘结剂 ${ }^{[105]}$

Figure 11 XG binder ${ }^{[105]}$

(a) Chemical structure of XG binder, (b) Concept transfer from macroscopic to nanoscopic world and structural analogy of millipede to that of native-XG towards strong adhesion. A series of small legs in the millipede corresponds to multiple short side chains in native-XG (both colored in red). (c) Electrochemical performance with different charged polymeric binders, the electrodes ( $\mathrm{Si}$ loading $=0.3 \mathrm{mg} \bullet \mathrm{cm}^{-2}$ ) were measured at $1 \mathrm{C}\left(3500 \mathrm{~mA} \cdot \mathrm{g}^{-1}\right)$ for both charge and discharge in the voltage range of $0.01 \sim 1.0 \mathrm{~V}$ vs. $\mathrm{Li} / \mathrm{Li}^{+}$

结剂制备出硅负载量为 $0.3 \mathrm{mg} \cdot \mathrm{cm}^{-2}$ 的硅复合电极, 并 在 $1 \mathrm{C}$ 下进行 200 次循环后, 仍保留有 $2150 \mathrm{mAh} \cdot \mathrm{g}^{-1}$ 的 比容量, 以及高达 $72.2 \%$ 的容量保持率，如图 $11 \mathrm{c}$ 所示， 远高于传统粘结剂, 证实 XG 作为新型天然粘结剂的潜 力.

除以上粘结剂外, 研究人员还制备出新型耐高温粘 结剂结冷胶 ${ }^{[106]}$, 含有蛋白质以增加柔韧性的粘结剂卡 拉亚胶 ${ }^{[107]}$, 骨架构象能在锂化过程中转变的果胶 ${ }^{[108]}$, 从肠膜明串珠菌细菌中提取的天然多糖右旋糖酩 ${ }^{[109]}$, 模仿贻贝螺旋连接的儿茶酚共聚物 ${ }^{[110]}$ 等粘结剂.

\section{5 小结}

新型生物高分子粘结剂大多受自然界启发, 直接从 自然界的天然聚合物中提取, 或者模拟出相似的生物特 性以获得优异的粘结剂. 生物高分子粘结剂主要都是由 多糖构成，根据不同的多糖成分分为海藻酸盐、壳聚糖、 $\beta$-环糊精与生物胶等多个种类. 生物高分子粘结剂虽然
具有可再生性与多样性等多重优点, 并且能模拟生物行 为, 部分在循环性能上也有了进一步的提升, 但是容量 的损失仍不可避免. 同时, 非活性材料的占比太重, 加 上导电添加剂的添加, 粘结剂本身不导电的特性, 都为 改善电池的循环与容量特性增加难度.

\section{4 导电聚合物粘结剂}

通常, 制备硅负极时, 需要导电添加剂, 例如乙炔 黑、碳黑等, 来确保高电导率. 但是大量导电剂的添加 会减少活性物质的质量比, 降低锂离子电池的储存比容 量. 同时, 导电添加剂没有粘结性能, 容易在体积膨胀 时从硅颗粒上脱离, 断开电连接, 降低电化学活性. 与 传统生物粘结剂相比, 导电聚合物粘结剂能在电极材料 的制作过程中具有粘结剂和导电添加剂的双重功能, 不 仅可以促进电池电化学过程中电子的快速传输, 解决传 统导电添加剂引起的电连接问题, 而且能够减少电极材 
料中非活性物质占比, 保持电池电化学性能的同时提升 电池容量. 在硅负极粘结剂的研究中, 导电聚合物因自 身优异的结构与化学特性, 受到大家广泛的关注.

\subsection{PFFOMB 粘结剂}

$\mathrm{Liu}$ 等 ${ }^{[11]}$ 开发出可在阴极还原环境下用于高电子 电导率的新型 $\mathrm{n}$ 型掺杂聚合物粘结剂聚( 9 , 9-二辛基芴共-樟脑酤-甲基苯甲酸)(PFFOMB), 如图 12a 所示, 其分 子结构基于聚芴(PF)型聚合物, 引入两个关键功能基团 羰基 $\mathrm{C}=\mathrm{O}$ 和甲基苯甲酸酯- $\mathrm{PhCOOCH}_{3}(\mathrm{MB})$ 来分别调节 最低空分子轨道(LUMO)电子状态和改善聚合物粘附性.

凭借实验设计的结构, PFFOMB 实现了优异的电子 传导性能和机械完整性, 与电极间实现紧密的电接触, 保持电极完整性, 从而获得高比容量和高稳定循环性 能, 如图 $12 \mathrm{~b}$ 所示, 在 650 次循环后比容量仍高达 2100 $\mathrm{mAh} \cdot \mathrm{g}^{-1}$, 容量损失率很小. 并且这种导电粘结剂的应 用成本很低，与目前的制造技术兼容，应用前景十分广 阔 ${ }^{[111]}$.

\subsection{PANI 粘结剂}

$\mathrm{Wu}$ 等 ${ }^{[112]}$ 通过原位聚合法, 利用植酸质子化聚苯胺 (PANI)上氮原子基团来制备导电聚合物, 并与纳米硅颗 粒混合, 组成良好的三维连接多孔网络结构. 复合电极 结构中, 植酸分子中磷酸基团可通过氢键与硅颗粒间进 行静电相互作用, 形成稳定的共价键连接, 改善因体积
变化引起的容量损失. 与 PAA, CMC 中羧酸基团性质类 似，这种相互作用会在聚合过程中与苯胺单体进一步交 联以产生保护导电涂层. 导电聚合物粘结剂的三维多孔 结构缩短有效扩散路径, 可有效促进电子和离子运输, 改善电极电化学性能. 同时, 这种粘结剂可通过简单的 溶液合成法进行制备，与传统涂层工艺相兼容，在商业 上应用潜力大. 实验 SEM 图可见, 2000 次电化学循环 后, 复合电极仍形成均匀且薄的 SEI 膜, 最终获得超过 5000 次的循环寿命与超过 90\%的高容量保持率，显示 出非常优异的循环稳定性. 随后, Zhao 等 ${ }^{[113]}$ 对复合电 极进行蒸汽碳化，也获得不俗的表现.

\subsection{PEDOT:PSS 粘结剂}

Higgins 等利用单个导电粘结剂聚 $(3,4-$ 亚乙基二氧 噻吩)/聚-(苯乙烯-4-磺酸盐)(PEDOT:PSS)制备出高硅含 量的硅负极, 结构如图 13a 所示. PEDOT:PSS 基硅负极 工作机制如图 13b 所示，不需要通过粘结剂来调节活性 材料和额外导电添加剂间的相互作用，消除锂化/去锂 化过程中硅和传统无机导电添加剂的物理分离导致的 容量损失, 获得均匀的电极结构, 如图 13c 所示, 提升 容量与循环特性. PEDOT:PSS 粘结剂电化学稳定性高、 机械强度高、制备成本低、具有水溶性, 通过简单的富 马酸 (FA) 原位二次掺杂处理可获得更加优异导电 性 ${ }^{[114,115]}$.
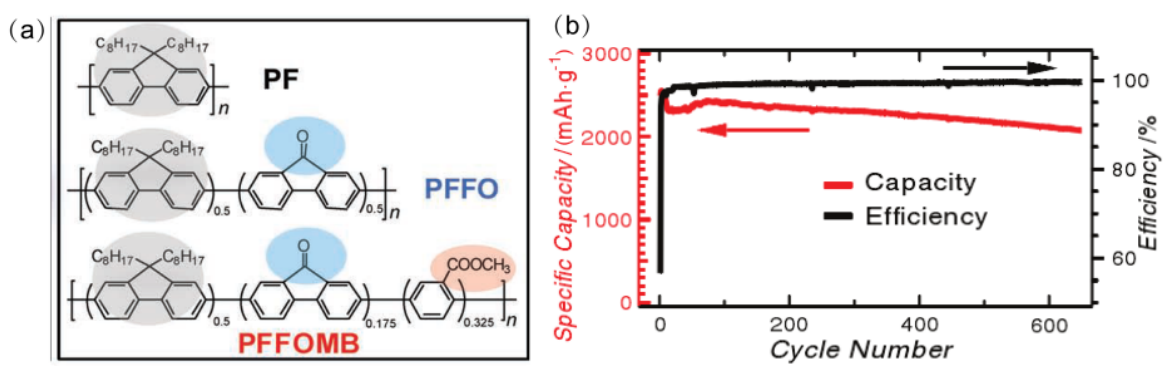

图 12 PFFPMB 导电粘结剂[111]

Figure 12 PFFPMB conductive binder ${ }^{[111]}$

(a) The molecular structure of the PF-type conductive polymers, with two key function groups in PFFOMB, carbonyl and methylbenzoic ester, for tailoring the conduction band and for improving the mechanical binding force, respectively. (b) Cycling performance of Si/PFFOMB electrode between a cycling voltage of $1 \mathrm{~V}$ and $0.01 \mathrm{~V}$ for over 650 cycles at $\mathrm{C} / 10$ rate

(a)

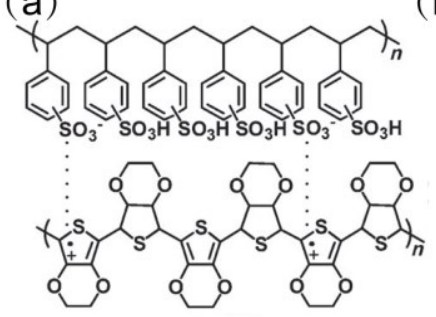

(b)

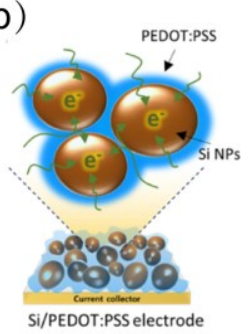

(c)

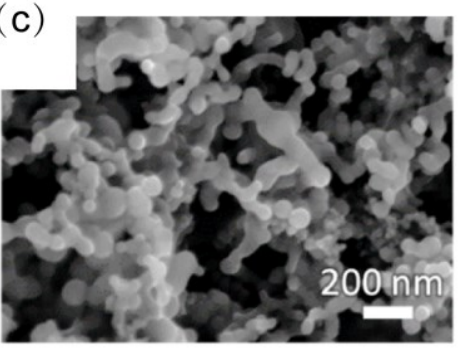

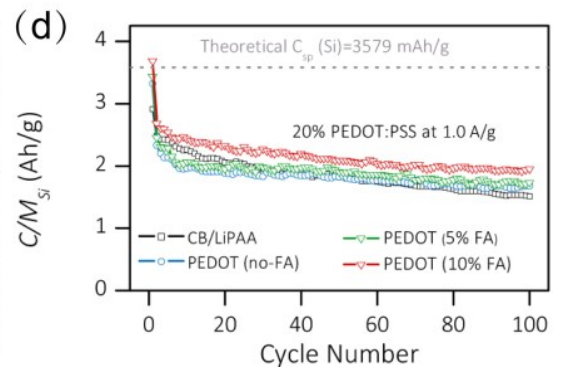

图 13 PEDOT:PSS 导电粘结剂

Figure 13 PEDOT:PSS conductive binder

(a) Structure of PEDOT:PSS, PEDOT:PSS/SiNP electrode ${ }^{[14]}$ : (b) working mechanism ${ }^{[115]}$, (c) the SEM image ${ }^{[15]}$ and (d) Cyclability of lithiation of these electrodes at $1 \mathrm{~A} \cdot \mathrm{g}^{-1}$ after initial cycling at $0.5 \mathrm{~A} \cdot \mathrm{g}^{-1[115]}$ 
凭借这些优异的性能, Higgins 等 ${ }^{[115]}$ 使用少量 PEDOT:PSS 进行原位次级掺杂处理, 可以制备出电导 率高达 $4.2 \mathrm{~S} \cdot \mathrm{cm}^{-1}$, 硅占比高达 $80 \mathrm{wt} \%$ 的高负载量硅负 极. 在 $1 \mathrm{mg} \cdot \mathrm{cm}^{-2}$ 的面负载量下, 复合硅负极初始循环 比容量高达 $3685 \mathrm{mAh} \cdot \mathrm{g}^{-1}$, 但首次循环效率约仅为 $78 \%$. 利用 FA 对复合电极进行二次掺杂处理, 如图 13d 所示, 当 FA 含量为 $10 \%$ 时, 获得优于 PAA 基硅负极的 循环特性. 这种涂覆性高、成本低的商业性硅负极制备 方式，在商业大规模生产上具有很高的应用前景.

在考虑 PEDOT:PSS 的高电子电导率的同时, 导电 粘结剂的离子电导率也会影响硅负极的电化学性能. 以 此为出发点, Zeng 等 ${ }^{[116]}$ 通过化学交联、化学还原和静电 自组装将离子导电聚环氧乙烷(PEO)和聚乙烯亚胺(PEI) 组装到高电导率的 PEDOT:PSS 链上来制备高性能交联 粘合剂(c-PEO-PEDOT:PSS/PEI). PEDOT:PSS 中的磺酸 盐和磺酸基团可以被改性以提供高离子导电性. 同时, PEO 和 PEI 聚合物链中丰富的孤电子对, 有利于锂离子 交换. 该聚合物粘合剂具有分别比 CMC 高出 14 和 90 倍的离子和电子传输性能, 同时显示出更强的速率性能 与循环稳定性. 在机械性能方面, PEDOT:PSS 和离子聚 合物间的交联和静电相互作用形成具有高模量的粘结 剂, 有助于保持电极完整性. c-PEO-PEDOT:PSS/PEI/Si 复合电极在 $1.0 \mathrm{~A} \cdot \mathrm{g}^{-1}$ 的电流密度下, 可获得 2440 $\mathrm{mAh} \cdot \mathrm{g}^{-1}$ 的初始循环比容量, 并且在 500 次循环后容量 保持在 $2027 \mathrm{mAh} \cdot \mathrm{g}^{-1}$.

Wang 等 ${ }^{[117]}$ 设计出一种具有高导电性, 优异的拉伸 性和延展性的新型水溶性可拉伸导电胶 $(\mathrm{CG})$ 聚合物粘 结剂. $\mathrm{CG}$ 粘结剂通过将 $d$-山梨糖醇和乙酸乙烯酯-丙烯 酸交联到导电聚合物聚(3,4-亚乙二氧基噻吩): 聚苯乙烯 磺酸盐(PEDOT:PSS)上制成, 可以拉伸至初始长度的 250\%和初始体积的 400\%而导电率基本不变. 硅颗粒上 的可拉伸导电胶涂层可以适应循环过程中活性 $\mathrm{Si}$ 颗粒 的大体积变化, 保持高结构完整性, 并在 $\mathrm{Si}$ 含量高达 $90 \%$ 的同时保持电极的高导电性, 在 700 次循环后在 $0.2 \mathrm{C}$ 下具有 $1500 \mathrm{mAh} \cdot \mathrm{g}^{-1}$ 的稳定容量, 具有 $80 \%$ 的高 初始库仑效率. 这种高度可拉伸和导电的聚合物粘合剂 在下一代高能与可拉伸电池中具有很高的应用价值.

\subsection{PPy 粘结剂与 PPyE 粘结剂}

$\mathrm{Liu}$ 等 ${ }^{[118]}$ 设计出三维新型硅负极, 利用单壁碳纳米 管 (SWCNTs) 作为电子强化剂, 将硅纳米颗粒(SiNP)包 覆在三维(3D)分层多孔纳米结构导电聚吡咯(PPy)粘结 剂框架中, 形成 SiNP/PPy/SWCNTs 三元复合硅负极. PPy 水凝胶粘结剂构成的三维分层多孔骨架, 能提供纳 米级孔隙 $(20 \sim 50 \mathrm{~nm})$, 促进电解质离子渗透, 改变扩散 通路和增加 $3 \mathrm{D}$ 互连性, 有效改善电子和离子传输性能, 显示出良好的电化学特性. 同时, 硅颗粒上带负电荷 $-\mathrm{OH}$ 基团和带正电荷聚吡咯聚合物主链间的静电效应 和植酸分子中磷酸基团与硅颗粒表面键合, 使 SWCNTs
与 $\mathrm{Si} / \mathrm{PPy}$ 骨架有效交织在一起, 并嵌入整个电极, 增加 电极完整性. 碳纳米管作为电子传导体, 提供连续的电 子传输网络和高孔隙率以适应硅颗粒的体积膨胀. 新型 的三维三元复合电极，实现超过 $1600 \mathrm{mAh} \cdot \mathrm{g}^{-1}$ 的可逆 放电比容量, 当电流密度为 $3.3 \mathrm{~A} \cdot \mathrm{g}^{-1}$ 时, 循环 1000 次 后，平均库伦效率达 $99.5 \%$, 极大地改善循环性能，表 现出长期稳定性. 这种先进的纳米结构导电粘结剂材料 可用于制备坚固耐用的高性能锂离子电池.

$\operatorname{PPyE}$ (聚(甲基丙烯酸-1-萠甲酯-甲基丙烯酸酯-共三氧化乙烯甲基醚甲基丙烯酸酯)与 PPy 类似，但含有 导电萠和三氧化二乙烯两种单元, 具有更好的粘附与导 电性, 改善电池性能. 获得的 PPyE 基硅负极在 0.15 $\mathrm{mg} \cdot \mathrm{cm}^{-2}$ 的面负载下, 以 $\mathrm{C} / 10$ 速率进行循环, 在 180 次 循环后，可获得 $2300 \mathrm{mAh} \cdot \mathrm{g}^{-1}$ 的比容量以及高达 $99.5 \%$ 的平均长期循环库仑效率, 优于 PPy 基硅负极 ${ }^{[19]}$.

\subsection{PFPQ-COONA 粘结剂}

锂离子充放电期间，粘合剂和硅间的电荷转移速率 取决于两个因素: 粘合剂中的电荷转移和粘合剂与活性 颗粒(例如 Si)之间的界面电荷转移速率. 因此好的导电 粘合剂在具有自身良好的导电性的同时，应能通过保持 与活性材料的接触来增强界面电荷转移. 最近, 为提高 导电粘结剂的电荷转移能力, Zhao 等 ${ }^{[120]}$ 合成一种新型 单组分导电粘合剂 PFPQ-COONa, 由具有良好粘结性 能的聚(9,9-双(3-丙酸)芴)(PF-COONa)骨架与 $10 \%$ 具有 优异速率性能的菲醌 (PQ) 基团组成. 一方面, PFPQ-COONa 和硅颗粒间形成的-COOSi-基团起到针定 点的作用, 有利于充放电过程中导电网络和 $\mathrm{Si}$ 颗粒间 充分接触, 大大缩短 $\mathrm{Si}$ 颗粒和聚合物链间的平均距离, 有利于界面处的电荷转移. 另一方面, PQ 中的羰基可以 在还原电位下还原成-O-Li 基团, 大大提高粘合剂导电 性. 除导电性外, PFPQ-COONa 的良好的机械性能也有 助于提升其循环稳定性. 这种方法为硅负极高性能导电 粘合剂的合理设计提供了新的视角: 良好的界面电荷转 移与导电粘合剂的高固有导电性同样重要, 可通过引入 具有所需性能的官能团来巧妙地调整粘结剂的性能, 最 终达到优异的循环稳定性.

\section{6 小结}

总之, 导电粘结剂因自身粘结性与导电性的双重属 性而受到广泛关注. 在进行导电粘结剂的设计时, 需同 时考虑粘结剂的离子电导率与电子电导率. 电子电导率 不仅受到粘结剂自身导电性的影响，还受到粘结剂与硅 颗粒间界面电子传导特性的影响. 同时, 粘结剂的机械 特性, 如拉伸性能、粘结性等都会对电池的性能有很大 的影响. 在选择导电粘结剂的时候, 应该综合考虑各种 性能, 以求达到最优的电池容量特性与循环稳定性. 部 分导电粘结剂可获得接近硅理论值的初始比容量, 但是 循环稳定性一般. 部分导电粘结剂能循环至 500 次后仍 具有 $1500 \mathrm{mAh} \cdot \mathrm{g}^{-1}$ 以上的比容量, 但是初始比容量较 
小. 目前, 如何找到一个有效的导电粘结剂同时能满足 高容量与高循环稳定性, 仍是一个巨大的挑战.

\section{5 其他粘结剂}

\section{1 聚乙烯醇(PVA)基粘结剂}

聚乙烯醇(PVA)中存在许多羟基, 与活性材料形成 强氢键, 这些强氢键使覆盖活性材料表面的粘结剂量增 加, 显著增强电极粘合强度. 同时, 高分子量 PVA 能通 过更长的聚合物链与活性材料间形成更多的絮凝结构, 在溶胀实验中吸收更少量电解质, 进一步增加粘合强 度, 保持电极完整性. Park 等 ${ }^{[121]}$ 通过两步聚合法制备出 高分子量聚乙烯醇(PVA)粘结剂, 在循环测试中, 高分 子量 PVA 粘结剂显示出比 PVDF 和 PAA 粘结剂更好的 循环性能.

Yook 等 ${ }^{[122]}$ 使用具有不同程度交联的聚乙烯醇-富 马酸(PVA-FA)作为聚合物粘结剂, 并应用于硅合金-石 墨复合电极. PVA 中羟基与 FA 中羧基间进行缩合反应, 如图 $14 \mathrm{~d}$ 所示, 并在 $150{ }^{\circ} \mathrm{C}$ 下酯化 $1 \mathrm{~h}$ 建立酯键, 形成 三维交联粘结剂. 粘结剂三维结构的建立与合金金属相 的存在, 减少在活性硅颗粒经过锂化/去锂化过程中产 生的应力, 有利于电子快速转移到硅, 提高电导率. 实 验证实, 在 $0.5 \mathrm{C}$ 的电流密度下, 使用 $\mathrm{PVA}$ 与 $5.0 \mathrm{wt} \% \mathrm{FA}$ 交联(PVA-5.0)粘结剂, 如图 14e 所示, 获得 $454.1 \mathrm{mAh} \cdot$ $\mathrm{g}^{-1}$ 的初始放电比容量以及 $98.6 \%$ 的库伦效率, 虽高于 石墨负极理论比容量, 但是远小于硅理论值, 也不能满 足商业需求.

此外, Liu 等 ${ }^{[123]}$ 通过原位热交联法, 利用水溶性聚 乙烯醇(PVA)和聚(乙烯亚胺)(PEI)合成低成本和环保的 新型聚合物粘结剂来提升硅负极电化学性能. PVA-PEI 粘结剂结构如图 14a 所示, 这种聚合物粘结剂含有着基 和亚氨基官能团，工作机制如图 14b 所示，能与硅颗粒
表面羟基进行共价连接，粘结性强，形成稳定可逆的互 连网络, 以及稳定的 SEI 膜, 增加粒子间接触性能, 有 利于电子转移, 改善电极电化学性能. PVA-PEI 基硅负 极在初始循环中表现出高达 $3072.9 \mathrm{mAh} \cdot \mathrm{g}^{-1}$ 的比容量, 如图 $14 \mathrm{c}$ 所示, 约为理论比容量的 $73.2 \%$, 初始库伦效 率为 $83.8 \%$, 在 300 次循环后具有 $1063.1 \mathrm{mAh} \cdot \mathrm{g}^{-1}$ 的比 容量, 虽容量损失很大, 但表现出长期循环的可能性.

\subsection{TBA-TEVS 交联粘结剂}

Jeena 等 ${ }^{[124]}$ 将新型共聚物聚(丙烯酸叔丁酯-共-三乙 氧基乙烯基硅烷)(TBA-TEVS), 作为粘结剂应用于硅负 极. TBA-TEVS 可通过简单的 2,2-偶氮二(异丁 腈)(AIBN)介导的自由基聚合合成，成本低且耗时少. 叔丁基能与羧酸官能团通过缩合反应形成高度互连的 $3 \mathrm{D}$ 网络, 并在 $220{ }^{\circ} \mathrm{C}$ 热处理时保护粘结剂网络不被破 坏, 减少由硅体积膨胀引起的电极崩解. 硅氧烷官能团 经数百个循环诱导形成稳定的薄 SEI 膜, 进一步稳定电 池性能. 实验通过改变 TBA 和 TEVS 摩尔比来合成一系 列无规共聚物，其中 $21 \%$ 摩尔比的 TBA-TEVS-21 在低 负载, 100 个循环内稳定性远优于其他比例的粘结剂. 高负载下 TBS-TEVS-21 粘结剂仍保持高达 2.02 $\mathrm{mAh} \cdot \mathrm{cm}^{-2}$ 的高面比容量, 50 次循环后仍高达 1.7 $\mathrm{mAh} \cdot \mathrm{cm}^{-2}$, 循环性能优于传统粘结剂, 进一步证实它 是一种十分有前景的粘结剂.

\section{3 聚酰亚胺(PI)粘结剂和聚酰胺酰亚胺(PAI)}

聚酰亚胺(PI), 一种著名的工程聚合物，因其优异 的综合性能，包括优异的力学性能，显著的化学热稳定 性和出色的耐溶剂性，已经在许多领域得到应用. 2013 年, 聚酰亚胺(PI)作为一种新型粘结剂, 被 $\mathrm{Kim}$ 等 ${ }^{[125]}$ 应 用于硅负极. 在锂化/去锂化过程中, PI 中 $\mathrm{C}=\mathrm{O}$ 基团能 与 $\mathrm{Li}^{+} 、 \mathrm{e}^{-}$发生反应, 提高电极比容量. 采用 $\mathrm{Ag}$ 辅助化 学蚀刻法制备的高比表面积 PI 基硅负极, 获得比 PVDF (a)

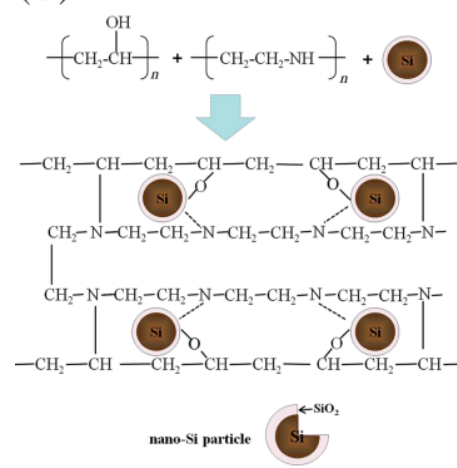

(b)

(c)
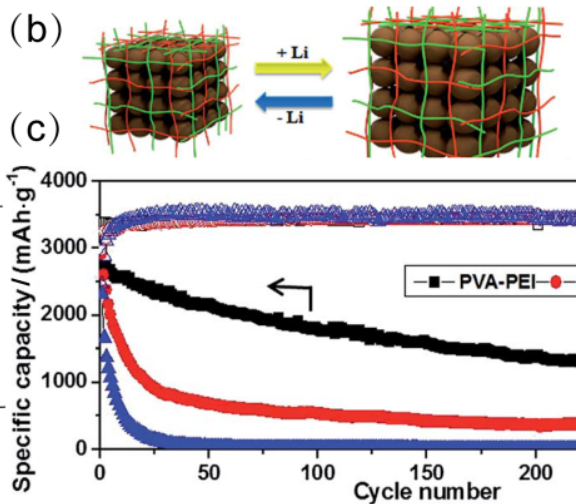

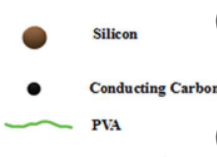

(d)

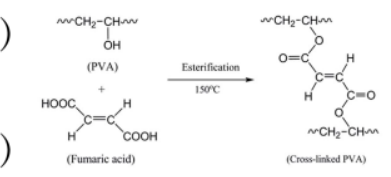

图 14 PVA-PEI 与 PVA-FA 粘结剂

Figure 14 PVA-PEI and PVA-FA binders

(a) The chemical structure and illustrative interaction between cross-linked PVA-PEI and silicon particles ${ }^{[123]}$, (b) the proposed working mechanism of PVA-PEI binder for silicon electrodes ${ }^{[123]}$, (c) the cycling performance and the coulombic efficiency of Si anodes with PVA-PEI and other binders ${ }^{[123]}$. (d) Schematic representation of the cross-linking of poly(vinylalcohol) (PVA) binders through esterification between PVA and fumaric acid ${ }^{[122]}$, (e) discharge capacities of the composite electrodes employing PVAs with different degrees of cross-linking $(0.5 \mathrm{C}$ constant current $(\mathrm{CC})$ and constant voltage $(\mathrm{CV})$ charge, $0.5 \mathrm{C} C \mathrm{C}$ discharge, cut-off voltage: $0.01 \sim 1.5 \mathrm{~V})^{[122]}$ 
更好的机械性能与恢复能力, 减小巨大体积变化引起的 容量损失, 改善电化学性能. 在 $200 \mathrm{~mA} \cdot \mathrm{g}^{-1}$ 的电流密度 下, PI/蚀刻硅复合电极能获得 $2184 \mathrm{mAh} \cdot \mathrm{g}^{-1}$ 的初始比容 量与 $80.7 \%$ 的首次库伦效率，远高于 PVDF 粘结剂基硅 负极. 此外, 可通过引入不同分子量的聚乙二醇(PEG) 链段来对 PI 进行改性. PI 的主要部分由偏苯三酸酐氯化 物(TMAC) 和 4,4'-亚甲基二苯胺制成, PEG 段通过与 TMAC 的酯化反应引入. 这种 PI 共聚物在被三乙胺中 和后获得优异的水溶性, 能作为水溶性粘合剂用于硅负 极, 并显著改善硅负极的电化学性能. 特别是对于 PI-200(聚酰亚胺与 PEG-200 共聚)硅基电极, 在 $0.1 \mathrm{C}$ 的 电流密度下, 其初始放电比容量高达 $2989.7 \mathrm{mAh} \cdot \mathrm{g}^{-1}$, 循环 200 次后保持在 $2235.5 \mathrm{mAh} \cdot \mathrm{g}^{-1}$, 实现了高比容量 和高循环稳定性. 结果表明, 改性聚酰亚胺具有较好的 机械性能和耐溶剂性, 以及较好的弹性和较高的拉伸强 度, 可以承受充放电循环过程中硅颗粒的体积变化, 防 止硅电极粉化, 最终提高硅负极电化学性能 ${ }^{[126]}$.

聚酰胺酰亚胺(PAI), 是具有优异的机械性能和热 稳定性能的共聚酰亚胺类的聚合物, 可通过低温溶液聚 合法获得. 2008 年, Choi 等 ${ }^{[127]}$ 将 PAI 聚合物作为粘结剂 应用于硅基负极, 并获得 74.9\%的初始库仑效率, 远高 于 PVDF 基硅负极. 经过 20 次循环后, Si-PAI 电极的放 电比容量为 $1700 \mathrm{mAh} \cdot \mathrm{g}^{-1}$, 显示出 PAI 作为粘结剂的优 良特性. 与 PVDF 相比, PAI 具有高达 $63.3 \mathrm{MPa}$ 的拉伸 强度和低膨胀率, 更有效地抑制充放电过程中活性硅材 料的体积膨胀, 有效地抑制电子传导网络的分解, 减少 比容量损失.

\section{4 共价交联聚丙烯酰胺(c-PAM)粘合剂}

$\mathrm{Zhu}$ 等 ${ }^{[128]}$ 合成出一种新型的共价交联聚丙烯酰胺 c-PAM 粘合剂, 具有高拉伸性和丰富的酰胺基团, 可用 于高容量硅负极. c-PAM 粘结剂的独特性在于其 3D 超 支化骨架结构和丰富的酰胺官能团, 可以通过这双重途 径增强硅负极的机械性能. 制备出的交联度为 $1 \%$ 的 c-PAM 粘结剂可以拉伸至其初始长度的 6.1 倍, 赋予硅 负极高的柔韧性和可折叠性. 强 3D 粘合剂网络不仅能 够连接核心电池组件并在循环过程中保持电极的完整 性, 从而显著提高硅负极的循环性能和倍率性能. 在 $0.1 \mathrm{C}$ 的电流密度下, Si/c-PAM-0.001 复合电极 100 次循 环后达到 $2843 \mathrm{mAh} \cdot \mathrm{g}^{-1}$ 的可逆容量, 和 $90.1 \%$ 的容量保 持率. 这种高弹性的粘结剂可以应用到其他大体积变化 的电极材料中.

\subsection{GO “双重作用” 粘结剂}

Shan 等 ${ }^{[129]}$ 首次报道将石墨烯氧化物(GO)作为新型 “双重作用”粘合剂应用于硅负极. GO 不仅能提供类石 墨烯的多孔 3D 框架, 在充放电期间调节硅颗粒的体积 变化, 同时与硅颗粒间可形成化学键. 这种独特的 GO 粘合剂的双重作用, 在保持其结构完整性的同时形成稳 定的固体电解质 (SEI)薄膜, 使制备出的复合 GO/SiNP
电极在第 50 次循环时显示出 $2400 \mathrm{mAh} \cdot \mathrm{g}^{-1}$ 的比容量, 在第 100 次循环时显示出 $2000 \mathrm{mAh} \cdot \mathrm{g}^{-1}$. 在进行倍率性 能测试时, 在 $2 \mathrm{~A}$ 时获得高达 $1009 \mathrm{mAh} \cdot \mathrm{g}^{-1}$ 的容量, 这 在目前为止是针对硅负极非常高的容量值. 水溶性粘结 剂 GO 具有环境友好性, 制备比较简单, 有望实现大规 模生产, 对新一代高性能锂电池开发有很好的借鉴作 用.

\section{$5.6 \mathrm{~N}$-掺杂聚丙烯腈(N-PAN)粘结剂}

Zhang 等 ${ }^{[130]}$ 利用热解的 N-掺杂聚丙烯腈(N-PAN) 粘结剂网络, 以改善硅负极的循环稳定性. 首先将亚微 米硅颗粒分散在石墨烯片中以改善电子传导并避免局 部聚集. 实验证明, PAN 在 $400{ }^{\circ} \mathrm{C}$ 下热解形成致密结构, 可实现整个电极的结构稳定性，并应该严格控制温度防 止 PAN 在更高温度下过度碳化形成刚性结构, 缺少拉 伸性, 导致复合电极的崩解. 热解的 $\mathrm{N}$ 掺杂粘合剂网络 中包封的 $\mathrm{Si}$ 颗粒可以在第 100 次循环时保持约 1700 $\mathrm{mAh} \cdot \mathrm{g}^{-1}$ 的放电容量, 与在第 20 次循环时完全丧失其容 量的单个 $\mathrm{Si}$ 颗粒形成鲜明的对比.

\section{6 结论与展望}

本文综合讨论近年来不同粘结剂在锂离子电池硅 基负极的应用, 并详述其特性. 在传统粘结剂中, 最先 发展起来的 PVDF 粘结剂因其溶剂对环境有害, 与活性 硅颗粒间弱范德华力连接，比容量损失迅速等不良特性 而急需寻找替代产品. CMC 与 PAA 都是水溶性粘结剂, 并且存在羧基, 能与硅表面 $\mathrm{SiO}_{2}$ 共价键和, 保持电极完 整性, 极大地改善电池循环特性. 但是线性聚合物的特 性使这几种聚合物在锂化/去锂化过程中容易形成裂纹、 粉化, 进而降低电化学性能. 三维共价交联聚合物, 如 CMC-CA, PAA-CMC, PAA-PVA 等的出现, 有效抑制硅 纳米颗粒的大的移动, 防止在循环过程中由硅复合电极 经历大体积变化的情况下破坏导电网络, 从而稳定电 极, 进一步提升循环稳定特性, 为新型粘结剂的商用提 供基础.

考虑环境友好性，随后发展的新型粘结剂多为生物 聚合物，在生物中可提取或直接模拟生物特性，如 Alg、 $\mathrm{CS} 、 \beta-\mathrm{CDp}$ 、生物胶等及其交联聚合物粘结剂，具有环 境友好性、丰富性、低成本、可再生性与多样性等多重 优异特性, 都表现出优异的比容量特性与循环稳定性, 为锂离子电池硅负极的发展提供另一种有效的方法.

通常, 在制备复合电极时, 需要加入大量导电添加 率来确保高电导率, 降低活性物质占比, 减小比容量, 并且容易脱落, 降低电化学活性. 而导电粘结剂, 如 PFFOMB, PANI, PEDOT:PSS 等聚合物粘结剂, 具有粘 结剂和导电添加剂的双重功能, 对比其他粘结剂, 解决 传统导电添加剂引起的导电问题, 并减少非活性物质在 电极中占比, 提升电池比容量. 除此之外, 还存在一些 少见的粘结剂, 如 PVA 基粘结剂等, 也在一定程度上对 
电池性能做出改进.

部分粘结剂的性能如表 1 所示, 现在能做到的较好 的粘结剂初始容量可达到 $3500 \mathrm{mAh} \cdot \mathrm{g}^{-1}$ 以上, 但是容 量在 100 次循环内很容易损失到 $80 \%$ 以下. 相比较而言, 部分交联的生物粘结剂与导电粘结剂能获得较好的容 量特性与循环特性.

在考虑粘结剂的特性时, 应考虑以下几点: (1)首先 要考虑粘结剂的最基本的粘结特性, 交联聚合物形成的 3D 结构能有效减少活性物质与导电添加剂的脱落, 帮 助稳定性能. (2)粘结剂自身的导电特性也很重要, 对于 导电粘结剂, 应该同时考虑粘结剂内部与各物质间的电 子电导率与离子电导率, 以保证电子与锂离子都能有效 地进行传输, 减少电池容量的损失. 对于其他粘结剂, 引入导电组分, 同样能增加整个电极的容量特性, 能减 少部分因为导电添加剂脱落引起的损失. (3)为增加粘结 剂的自修复特性, 在考虑利用胺基、羧基等形成氢键的 同时, 可同时考虑增加离子键, 进一步稳定结构. (4)电 解质的分解会使电解质成分改变, 影响离子传输性能, 并且产生有害气体, 在设计粘结剂时, 应考虑到这点.
(5)最后，部分电极中导电添加剂的存在，不仅减少活性 材料的占比，同时它的脱落对电极的导电性能也会产生 巨大的影响, 在设计粘结剂时, 考虑到粘结剂与硅材料 的共价连接的同时，应该考虑到与导电添加剂的紧密共 价连接, 进一步改善循环特性.

总之, 利用粘结剂能有效保持整个电极的接触完整 性, 减少活性物质的脱落与粉碎, 使电池始终保持较高 的比容量与较优异的循环特性, 为锂离子电池硅负极的 商用化提供切实有效的解决方案. 但是到目前为止, 大 多数粘结剂容量特性与循环特性上并不能做到兼容. 在 以后的粘结剂中，应同时考虑到 3D 结构、导电特性、 自修复特性、电解质分解与导电添加剂的影响, 使粘结 剂更有效地缓解硅在充放电过程中的体积膨胀与性能 改变, 以获得更优异的循环性能, 使未来的锂硅电池商 用化成为可能. 同时, 优异的粘结剂不仅能用于锂硅电 池行业，也可以向着绿色化、可穿戴电池等方向发展， 而对于其他体积变化巨大的储能电池领域，也将是一种 可行的改性方式.

表 1 部分粘结剂性能对比图

Table 1 Performance of different binders

\begin{tabular}{|c|c|c|c|c|}
\hline 粘结剂 & 特征 & 初始比容量 $/\left(\mathrm{mAh} \cdot \mathrm{g}^{-1}\right)$ & $\begin{array}{c}\text { 循环性能 } \\
\text { (循环次数/剩余比容量) }\end{array}$ & 参考文献 \\
\hline PVDF & 线性、水溶性、范德华力连接 & ca. 1000 & $50 \mathrm{th} / 715.6 \mathrm{mAh} \cdot \mathrm{g}^{-1}$ & [54] \\
\hline $\mathrm{CMC}$ & 共价连接、高刚性、水溶性 & ca. 4000 & $100 \mathrm{th} / 1000 \mathrm{mAh} \cdot \mathrm{g}^{-1}$ & [37] \\
\hline $\mathrm{CMC}-\mathrm{CA}$ & 交联、低成本、可用于大电流器件 & ca. 850 & $1000 \mathrm{th} / 720 \mathrm{mAh} \cdot \mathrm{g}^{-1}$ & [67] \\
\hline PAA & 丰富的羧基、水溶性 & ca. 3000 & $100 \mathrm{th} / 2400 \mathrm{mAh} \cdot \mathrm{g}^{-1}$ & {$[47]$} \\
\hline PAA-PVA & 三维交联 & ca. 3616 & $100 \mathrm{th} / 2283 \mathrm{mAh} \cdot \mathrm{g}^{-1}$ & {$[78]$} \\
\hline $\mathrm{Ca}^{2+}$-Alg & 离子介导 & ca. 2200 & $120 \mathrm{th} / 1822 \mathrm{mAh} \cdot \mathrm{g}^{-1}$ & [85] \\
\hline reDNA-Alg & 两亲性 & ca. 2500 & $160 \mathrm{th} / 2000 \mathrm{mAh} \cdot \mathrm{g}^{-1}$ & [90] \\
\hline CS-TPP & 三维交联 & ca. 2100 & $500 \mathrm{th} / 1722 \mathrm{mAh} \cdot \mathrm{g}^{-1}$ & [93] \\
\hline CS-ENR & 高粘性、高弹性 & ca. 3500 & $500 \mathrm{th} / 2310 \mathrm{mAh} \cdot \mathrm{g}^{-1}$ & [96] \\
\hline$\beta$-CDp-6AD & 动态交联 & ca. 1800 & $150 \mathrm{th} / 1640 \mathrm{mAh} \cdot \mathrm{g}^{-1}$ & [99] \\
\hline GG & 多糖聚合物 & ca. 3364 & $300 \mathrm{th} / 1561 \mathrm{mAh} \cdot \mathrm{g}^{-1}$ & [102] \\
\hline XG & 双螺旋超结构 & ca. 3000 & $200 \mathrm{th} / 2150 \mathrm{mAh} \cdot \mathrm{g}^{-1}$ & [105] \\
\hline c-PEO-PEDO:PSS/PEI & 高电子电导率与离子电导率 & 2440 & $500 \mathrm{th} / 2027 \mathrm{mAh} \cdot \mathrm{g}^{-1}$ & [117] \\
\hline PFPQ-COONA & 高界面电荷转移速率 & ca. 3600 & $100 \mathrm{th} / 2400 \mathrm{mAh} \cdot \mathrm{g}^{-1}$ & [121] \\
\hline PVA-PEI & 共价连接、水溶性 & 3072.9 & 300th $/ 1063.1 \mathrm{mAh} \cdot \mathrm{g}^{-1}$ & [124] \\
\hline c-PAM & 高拉伸性、丰富的酰胺基团 & ca. 3160 & $100 \mathrm{th} / 2843 \mathrm{mAh} \cdot \mathrm{g}^{-1}$ & [129] \\
\hline
\end{tabular}

\section{作者简介}

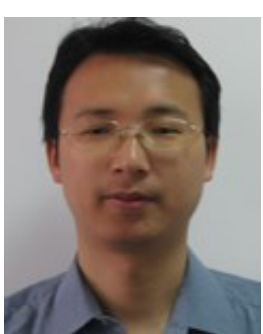

魏良明, 男, 1974 年生, 研究员. 2004 年在中科院长春应用 化学研究所获得高分子化学与物理专业博士学位, 同年进入 上海交通大学电子信息与电气工程学院微纳电子学系工作至 今. 主要从事新一代高性能锂离子电池/超级电容器以及传感 器的研究.

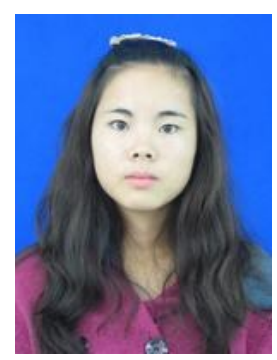

王晓钰, 女, 1995 年生, 硕士生. 2013 年于电子科技大学 微电子与固体电子学院攻读学士学位, 2017 年进入上海交通 大学电子信息与电气工程学院微纳电子学系攻读硕士学位, 主要进行锂离子电池与电池粘结剂的研究. 


\section{References}

[1] Armand, M.; Tarascon, J. M. Nature 2008, 451, 652.

[2] Tarascon, J. M.; Armand, M. Nature 2001, 414, 359.

[3] Etacheri, V.; Marom, R.; Elazari, R.; Salitra, G.; Aurbach, D. Energ Environ. Sci. 2011, 4, 3243.

[4] Huggins, R. A.; Boukamp, B. A. J. Electrochem. Soc. 1984, 128, 725 .

[5] Li, J.; Yang, C.; Zhang, J.; Zhang, X.; Xia, B. Acta Chim. Sinica 2010, 68, 646 (in Chinese). (李佳, 杨传铮, 张建, 张熙贵, 夏保 佳, 化学学报, 2010, 68, 646.)

[6] Zhao, L. J.; Zhao, Q.; Niu, Z. Q.; Liang, J.; Tao, Z. L.; Chen, J. Chinese J. Inorg. Chem. 2016, 32, 929.

[7] Aricò, A. S.; Bruce, P.; Scrosati, B.; Tarascon, J.; Schalkwijk, W. V. Nat. Mater. 2005, 4, 366.

[8] Sharma, R. A. J. Electrochem. Soc. 1976, 123, 1763.

[9] Seefurth, R. N.; Sharma, R. A. J. Electrochem. Soc. 1977, 124, 1207.

[10] Hatchard, T. D.; Dahn, J. R. J. Electrochem. Soc. 2004, 151, A838.

[11] Deshpande, R.; Cheng, Y.-T.; Verbrugge, M. W. J. Power Sources 2010, 195, 5081 .

[12] Kamali, A. R.; Fray, D. J. J. New Mater. Electrochem. Syst. 2010, 13, 147.

[13] Park, C. M.; Kim, J. H.; Kim, H.; Sohn, H. J. Chem. Soc. Rev. 2010 , 39,3115

[14] Zhang, W.-J. J. Power Sources 2011, 196, 13.

[15] Wu, H.; Cui, Y. Nano Today 2012, 7, 414.

[16] Park, M. H.; Kim, M. G.; Joo, J.; Kim, K.; Kim, J.; Ahn, S.; Cui, Y.; Cho, J. Nano Lett. 2009, 9, 3844.

[17] Song, T.; Xia, J.; Lee, J. H.; Lee, D. H.; Kwon, M. S.; Choi, J. M.; Wu, J.; Doo, S. K.; Chang, H.; Park, W. I.; Zang, D. S.; Kim, H.; Huang, Y.; Hwang, K. C.; Rogers, J. A.; Paik, U. Nano Lett. 2010, 10,1710 .

[18] Wu, H.; Chan, G.; Choi, J. W.; Ryu, I.; Yao, Y.; McDowell, M. T.; Lee, S. W.; Jackson, A.; Yang, Y.; Hu, L.; Cui, Y. Nat. Nanotechnol. 2012, 7, 310 .

[19] Chen, W.; Lei, T.; Qian, T.; Lv, W.; He, W.; Wu, C.; Liu, X.; Liu, J.; Chen, B.; Yan, C.; Xiong, J. Adv. Energy Mater. 2018, 8, 1702889.

[20] Shi, F.; Song, Z.; Ross, P. N.; Somorjai, G. A.; Ritchie, R. O.; Komvopoulos, K. Nat. Commun. 2016, 7, 11886.

[21] Choi, S.; Kwon, T. W.; Coskun, A.; Choi, J. W. Science 2017, 357, 279.

[22] Ryu, J. H.; Kim, J. W.; Sung, Y.-E.; Oh, S. M. Electrochem. Solid-State Lett. 2004, 7, A306.

[23] Beaulieu, L. Y.; Eberman, K. W.; Turner, R. L.; Krause, L. J.; Dahn, J. R. Electrochem. Solid-State Lett. 2001, 4, A137.

[24] Timmons, A.; Dahn, J. R. J. Electrochem. Soc. 2006, 153, A1206.

[25] Verbrugge, M. W.; Cheng, Y.-T. J. Electrochem. Soc. 2009, 156, A927.

[26] Wu, H.; Zheng, G.; Liu, N.; Carney, T. J.; Yang, Y.; Cui, Y. Nano Lett. 2012, 12, 904.

[27] Yen, Y.-C.; Chao, S.-C.; Wu, H.-C.; Wu, N.-L. J. Electrochem. Soc. 2009, 156, A95.

[28] Feng, K.; Li, M.; Liu, W.; Kashkooli, A. G.; Xiao, X.; Cai, M.; Chen, Z. Small 2018, 14, 1702737.

[29] Peng, B.; Cheng, F.; Tao, Z.; Chen, J. J. Chem. Phys. 2010, 133, 034701 .

[30] Wilson, A. M.; Dahn, J. R. J. Electrochem. Soc. 1995, 142, 326.

[31] Yang, J.; Winter, M.; Besenhard, J. O. Solid State Ionics 1996, 90 , 281.

[32] Ma, H.; Cheng, F.; Chen, J. Y.; Zhao, J. Z.; Li, C. S.; Tao, Z. L.; Liang, J. Adv. Mater. 2007, 19, 4067.

[33] Kang, K.; Song, K.; Heo, H.; Yoo, S.; Kim, G.-S.; Lee, G.; Kang, Y.-M.; Jo, M.-H. Chem. Sci. 2011, 2, 1090.

[34] Hu, L.; Wu, H.; Hong, S. S.; Cui, L.; McDonough, J. R.; Bohy, S.; Cui, Y. Chem. Commun. (Camb) 2011, 47, 367.

[35] Ren, W.; Wang, C.; Lu, L.; Li, D.; Cheng, C.; Liu, J. J. Mater. Chem. A 2013, 1, 13433.

[36] Hong, L.; Huang, X.; Chen, L.; Zhou, G.; Zhang, Z.; Yu, D.; Yu, J. M.; Ning, P. Solid State Ionics 2000, 135, 181.

[37] Bridel, J. S.; Azaïs, T.; Morcrette, M.; Tarascon, J. M.; Larcher, D. Chem. Mater. 2010, 22, 1229.

[38] Du, J.; Lin, N.; Qian, Y. Acta Chim. Sinica 2017, 75, 147 (in Chinese). (杜进, 林宁, 钱逸泰, 化学学报, 2017, 75, 147.)

[39] Anani, A.; Huggins, R. A. J. Power Sources 1992, 38, 351.

[40] Courtney, I. A.; Dahn, J. R. J. Electrochem. Soc. 1997, 144, 2045.
[41] Wilson, A. M.; Way, B. M.; Dahn, J. R.; van Buuren, T. J. Appl. Phys. 1995, 77, 2363.

[42] Saint, J.; Morcrette, M.; Larcher, D.; Laffont, L.; Beattie, S.; Pérès, J. P.; Talaga, D.; Couzi, M.; Tarascon, J. M. Adv. Funct. Mater. 2007, $17,1765$.

[43] Zhang, Y.; Zhu, Y.; Fu, L.; Meng, J.; Yu, N.; Wang, J.; Wu, Y. Chin. J. Chem. 2017, 35, 21.

[44] Kong, L.; Zhou, X.; Fan, S.; Li, Z.; Gu, Z. Acta Chim. Sinica 2016 74,620 (in Chinese). (孔丽娟, 周晓燕, 范赛英, 李在均, 顾志国, 化学学报, 2016, 74, 620.)

[45] Chen, Z.; Christensen, L.; Dahn, J. R. Electrochem. Commun. 2003 5,919 .

[46] Li, J.-T.; Wu, Z.-Y.; Lu, Y.-Q.; Zhou, Y.; Huang, Q.-S.; Huang, L.; Sun, S.-G. Adv. Energy Mater. 2017, 7, 1701185.

[47] Magasinski, A.; Zdyrko, B.; Kovalenko, I.; Hertzberg, B.; Burtovyy, R.; Huebner, C. F.; Fuller, T. F.; Luzinov, I.; Yushin, G. ACS Appl. Mater. Interfaces 2010, 2, 3004.

[48] Du, L.; Zhuang, Q.; Wei, T.; Shi, Y.; Qiang, Y.; Sun, S. Acta Chim. Sinica 2011, 69, 2641 (in Chinese). (杜莉莉, 庄全超, 魏涛, 史月 丽, 强颖怀, 孙世刚, 化学学报, 2011, 69, 2641.)

[49] Hochgatterer, N. S.; Schweiger, M. R.; Koller, S.; Raimann, P. R.; Wöhrle, T.; Wurm, C.; Winter, M. Electrochem. Solid-State Lett. 2008, $11, \mathrm{~A} 76$.

[50] Kang, Y.-M.; Go, J.-Y.; Lee, S.-M.; Choi, W.-U. Electrochem Commun. 2007, 9, 1276.

[51] Kim, I.-s.; Blomgren, G. E.; Kumta, P. N. Electrochem. Solid-State Lett. 2004, 7, A44.

[52] Kim, I.-S.; Kumta, P. N. J. Power Sources 2004, 136, 145.

[53] Li, J.; Christensen, L.; Obrovac, M. N.; Hewitt, K. C.; Dahn, J. R. J. Electrochem. Soc. 2008, 155, A234.

[54] Xu, Y.; Yin, G.; Ma, Y.; Zuo, P.; Cheng, X. J. Power Sources 2010, 195, 2069.

[55] Santimetaneedol, A.; Tripuraneni, R.; Chester, S. A.; Nadimpalli, S P. V. J. Power Sources 2016, 332, 118.

[56] Grillet, A. M.; Humplik, T.; Stirrup, E. K.; Roberts, S. A.; Barringer, D. A.; Snyder, C. M.; Janvrin, M. R.; Apblett, C. A. J. Electrochem. Soc. 2016, 163, A 1859

[57] Drofenik, J.; Gaberscek, M.; Dominko, R.; Poulsen, F. W.; Mogensen, M.; Pejovnik, S.; Jamnik, J. Electrochim. Acta 2003, 48, 883.

[58] Lestriez, B.; Bahri, S.; Sandu, I.; Roue, L.; Guyomard, D. Electrochem. Commun. 2007, 9, 2801

[59] Li, J.; Lewis, R. B.; Dahn, J. R. Electrochem. Solid-State Lett. 2007, 10, A17.

[60] Mazouzi, D.; Lestriez, B.; Roué, L.; Guyomard, D. Electrochem Solid-State Lett. 2009, 12, A215.

[61] Chen, L.; Xie, X.; Xie, J.; Wang, K.; Yang, J. J. Appl. Electrochem. 2006, 36, 1099.

[62] Beattie, S. D.; Larcher, D.; Morcrette, M.; Simon, B.; Tarascon, J. M. J. Electrochem. Soc. 2008, 155, A158.

[63] Nguyen, C. C.; Yoon, T.; Seo, D. M.; Guduru, P.; Lucht, B. L. ACS Appl. Mater. Interfaces 2016, 8, 12211.

[64] Buqa, H.; Holzapfel, M.; Krumeich, F.; Veit, C.; Novák, P. J. Power Sources 2006, 161, 617.

[65] Lee, K.; Lim, S.; Go, N.; Kim, J.; Mun, J.; Kim, T. H. Sci. Rep. 2018, 8,11322

[66] Liu, Y.; Tai, Z.; Zhou, T.; Sencadas, V.; Zhang, J.; Zhang, L.; Konstantinov, K.; Guo, Z.; Liu, H. K. Adv. Mater. 2017, 29, 1703028.

[67] Karkar, Z.; Guyomard, D.; Roué, L.; Lestriez, B. Electrochim. Acta 2017, 258, 453

[68] Hays, K. A.; Ruther, R. E.; Kukay, A. J.; Cao, P.; Saito, T.; Wood, D. L.; Li, J. J. Power Sources 2018, 384, 136.

[69] Yabuuchi, N.; Shimomura, K.; Shimbe, Y.; Ozeki, T.; Son, J.-Y.; Oji, H.; Katayama, Y.; Miura, T.; Komaba, S. Adv. Energy Mater. 2011, 1,759 .

[70] Han, Z.-J.; Yabuuchi, N.; Shimomura, K.; Murase, M.; Yui, H.; Komaba, S. Energ. Environ. Sci. 2012, 5, 9014.

[71] Han, Z. J.; Yamagiwa, K.; Yabuuchi, N.; Son, J. Y.; Cui, Y. T.; Oji, H.; Kogure, A.; Harada, T.; Ishikawa, S.; Aoki, Y.; Komaba, S. Phys. Chem. Chem. Phys. 2015, 17, 3783.

[72] Komaba, S.; Yabuuchi, N.; Ozeki, T.; Han, Z.-J.; Shimomura, K.; Yui, H.; Katayama, Y.; Miura, T. J. Phys. Chem. C 2011, 116, 1380.

[73] Komaba, S.; Shimomura, K.; Yabuuchi, N.; Ozeki, T.; Yui, H.; Konno, K. J. Phys. Chem. C 2011, 115, 13487.

[74] Kang, S.; Yang, K.; White, S. R.; Sottos, N. R. Adv. Energy Mater. 
2017, 7, 1700045 .

[75] Koo, B.; Kim, H.; Cho, Y.; Lee, K. T.; Choi, N. S.; Cho, J. Angew. Chem. Int. Ed. Engl. 2012, 51, 8762.

[76] Wei, L.; Chen, C.; Hou, Z.; Wei, H. Sci. Rep. 2016, 6, 19583.

[77] Song, J.; Zhou, M.; Yi, R.; Xu, T.; Gordin, M. L.; Tang, D.; Yu, Z.; Regula, M.; Wang, D. Adv. Funct. Mater. 2014, 24, 5904.

[78] Lee, K.; Lim, S.; Tron, A.; Mun, J.; Kim, Y.-J.; Yim, T.; Kim, T.-H. RSC Adv. 2016, 6, 101622.

[79] Lee, S.-Y.; Choi, Y.; Hong, K.-S.; Lee, J. K.; Kim, J.-Y.; Bae, J.-S.; Jeong, E. D. Appl. Surf. Sci. 2018, 447, 442.

[80] Zhang, G.; Yang, Y.; Chen, Y.; Huang, J.; Zhang, T.; Zeng, H.; Wang, C.; Liu, G.; Deng, Y. Small 2018, e1801189.

[81] Lü, L.; Lou, H.; Xiao, Y.; Zhang, G.; Wang, C.; Deng, Y. RSC Adv. 2018, 8, 4604.

[82] Lim, S.; Lee, K.; Shin, I.; Tron, A.; Mun, J.; Yim, T.; Kim, T.-H. J. Power Sources 2017, 360, 585.

[83] Kovalenko, I.; Zdyrko, B.; Magasinski, A.; Hertzberg, B.; Milicev, Z.; Burtovyy, R.; Luzinov, I.; Yushin, G. Science 2011, 334, 75.

[84] Liu, J.; Zhang, Q.; Wu, Z. Y.; Wu, J. H.; Li, J. T.; Huang, L.; Sun, S. G. Chem. Commun. (Camb) 2014, 50, 6386.

[85] Yoon, J.; Oh, D. X.; Jo, C.; Lee, J.; Hwang, D. S. Phys. Chem. Chem. Phys. 2014, 16, 25628.

[86] Zhang, L.; Zhang, L.; Chai, L.; Xue, P.; Hao, W.; Zheng, H. J. Mater. Chem. A 2014, 2, 19036.

[87] Gu, Y.; Yang, S.; Zhu, G.; Yuan, Y.; Qu, Q.; Wang, Y.; Zheng, H. Electrochim. Acta 2018, 269, 405.

[88] Wu, Z.-Y.; Deng, L.; Li, J.-T.; Huang, Q.-S.; Lu, Y.-Q.; Liu, J.; Zhang, T.; Huang, L.; Sun, S.-G. Electrochim. Acta 2017, 245, 371.

[89] Kim, S.; Jeong, Y. K.; Wang, Y.; Lee, H.; Choi, J. W. Adv. Mater. 2018, 30, e1707594.

[90] Kong, L. J.; Li, R. Y.; Yang, Y. Q.; Li, Z. J. RSC Adv. 2016, 6, 76344.

[91] Gendensuren, B.; Oh, E.-S. J. Power Sources 2018, 384, 379.

[92] Ibezim, E. C.; Andrade, C. T.; Marcia, C.; Barretto, B.; Odimegwu, D. C.; Lima, F. F. D. Ibnosina J Med BS. 2011, 3, 77.

[93] Yue, L.; Zhang, L.; Zhong, H. J. Power Sources 2014, 247, 327.

[94] Wu, Z.-H.; Yang, J.-Y.; Yu, B.; Shi, B.-M.; Zhao, C.-R.; Yu, Z.-L. Rare Metals 2016.

[95] Lee, S. H.; Lee, J. H.; Nam, D. H.; Cho, M.; Kim, J.; Chanthad, C.; Lee, Y. ACS Appl. Mater. Interfaces 2018, 10, 16449.

[96] Biwer, A.; Antranikian, G.; Heinzle, E. Appl. Microbiol. Biotechnol. 2002, 59, 609.

[97] Jeong, Y. K.; Kwon, T. W.; Lee, I.; Kim, T. S.; Coskun, A.; Choi, J. W. Nano Lett. 2014, 14, 864.

[98] Kwon, T. W.; Jeong, Y. K.; Deniz, E.; Alqaradawi, S. Y.; Choi, J. W.; Coskun, A. ACS Nano 2015, 9, 11317.

[99] Ling, M.; Xu, Y.; Zhao, H.; Gu, X.; Qiu, J.; Li, S.; Wu, M.; Song, X.; Yan, C.; Liu, G.; Zhang, S. Nano Energy 2015, 12, 178.

[100] Ling, M.; Zhao, H.; Xiao, X.; Shi, F.; Wu, M.; Qiu, J.; Li, S.; Song, X.; Liu, G.; Zhang, S. J. Mater. Chem. A 2015, 3, 2036.

[101] Liu, J.; Zhang, Q.; Zhang, T.; Li, J.-T.; Huang, L.; Sun, S.-G. Adv. Funct. Mater. 2015, 25, 3599 .

[102] Dufficy, M. K.; Khan, S. A.; Fedkiw, P. S. J. Mater. Chem. A 2015, 3,12023 .

[103] Kuruba, R.; Datta, M. K.; Damodaran, K.; Jampani, P. H.; Gattu, B.; Patel, P. P.; Shanthi, P. M.; Damle, S.; Kumta, P. N. J. Power Sources 2015, 298, 331.
[104] Chen, D.; Yi, R.; Chen, S.; Xu, T.; Gordin, M. L.; Wang, D. Solid State Ionics 2014, 254, 65.

[105] Jeong, Y. K.; Kwon, T.-w.; Lee, I.; Kim, T.-S.; Coskun, A.; Choi, J. W. Energ. Environ. Sci. 2015, 8, 1224

[106] Klamor, S.; Schroder, M.; Brunklaus, G.; Niehoff, P.; Berkemeier, F.; Schappacher, F. M.; Winter, M. Phys. Chem. Chem. Phys. 2015, 17 5632.

[107] Bie, Y.; Yang, J.; Nuli, Y.; Wang, J. J. Mater. Chem. A 2017, 5, 1919.

[108] Yoon, D. E.; Hwang, C.; Kang, N. R.; Lee, U.; Ahn, D.; Kim, J. Y.; Song, H. K. ACS Appl. Mater. Interfaces 2016, 8, 4042.

[109] Zhao, X.; Yim, C.-H.; Du, N.; Abu-Lebdeh, Y. Ind. Eng. Chem. Res. 2018, 57, 9062 .

[110] Zhao, H.; Wei, Y.; Wang, C.; Qiao, R.; Yang, W.; Messersmith, P. B.; Liu, G. ACS Appl. Mater. Interfaces 2018, 10, 5440.

[111] Liu, G.; Xun, S.; Vukmirovic, N.; Song, X.; Olalde-Velasco, P.; Zheng, H.; Battaglia, V. S.; Wang, L.; Yang, W. Adv. Mater. 2011, 23, 4679 .

[112] Wu, H.; Yu, G.; Pan, L.; Liu, N.; McDowell, M. T.; Bao, Z.; Cui, Y Nat. Commun. 2013, 4, 1943.

[113] Zhao, S.; Yao, C.; Sun, L.; Xian, X. Ionics 2017, 24, 1039.

[114] Shao, D.; Zhong, H.; Zhang, L. ChemElectroChem 2014, 1, 1679.

[115] Higgins, T. M.; Park, S. H.; King, P. J.; Zhang, C. J.; McEvoy, N.; Berner, N. C.; Daly, D.; Shmeliov, A.; Khan, U.; Duesberg, G. Nicolosi, V.; Coleman, J. N. ACS Nano 2016, 10, 3702.

[116] Zeng, W.; Wang, L.; Peng, X.; Liu, T.; Jiang, Y.; Qin, F.; Hu, L.; Chu, P. K.; Huo, K.; Zhou, Y. Adv. Energy Mater. 2018, 8, 1702314.

[117] Wang, L.; Liu, T.; Peng, X.; Zeng, W.; Jin, Z.; Tian, W.; Gao, B.; Zhou, Y.; Chu, P. K.; Huo, K. Adv. Funct. Mater. 2018, 28, 1704858.

[118] Liu, B.; Soares, P.; Checkles, C.; Zhao, Y.; Yu, G. Nano Lett. 2013, 13,3414

[119] Park, S. J.; Zhao, H.; Ai, G.; Wang, C.; Song, X.; Yuca, N.; Battaglia, V. S.; Yang, W.; Liu, G. J. Am. Chem. Soc. 2015, 137, 2565.

[120] Zhao, Y.; Yang, L.; Zuo, Y.; Song, Z.; Liu, F.; Li, K.; Pan, F. ACS Appl. Mater. Interfaces 2018, 10, 27795.

[121] Park, H.-K.; Kong, B.-S.; Oh, E.-S. Electrochem. Commun. 2011, 13,1051 .

[122] Yook, S.-H.; Kim, S.-H.; Park, C.-H.; Kim, D.-W. RSC Adv. 2016, 6, 83126.

[123] Liu, Z.; Han, S.; Xu, C.; Luo, Y.; Peng, N.; Qin, C.; Zhou, M.; Wang, W.; Chen, L.; Okada, S. RSC Adv. 2016, 6, 68371.

[124] Jeena, M. T.; Bok, T.; Kim, S. H.; Park, S.; Kim, J. Y.; Park, S.; Ryu, J. H. Nanoscale 2016, 8, 9245.

[125] Kim, J. S.; Choi, W.; Cho, K. Y.; Byun, D.; Lim, J.; Lee, J. K. J. Power Sources 2013, 244, 521.

[126] Yao, D.; Yang, Y.; Deng, Y.; Wang, C. J. Power Sources 2018, 379, 26.

[127] Choi, N.-S.; Yew, K. H.; Choi, W.-U.; Kim, S.-S. J. Power Sources 2008, 177,590

[128] Zhu, X.; Zhang, F.; Zhang, L.; Zhang, L.; Song, Y.; Jiang, T.; Sayed, S.; Lu, C.; Wang, X.; Sun, J.; Liu, Z. Adv. Funct. Mater. 2018, 28 , 1705015.

[129] Shan, C.; Wu, K.; Yen, H. J.; Narvaez Villarrubia, C.; Nakotte, T.; Bo, X.; Zhou, M.; Wu, G.; Wang, H. L. ACS Appl. Mater. Interfaces 2018, 10, 15665.

[130] Zhang, Z.; Jiang, Y.; Peng, Z.; Yang, S.; Lin, H.; Liu, M.; Wang, D. ACS Appl. Mater. Interfaces 2017, 9, 32775. 